Review

\title{
A review on removing pharmaceutical contaminants from wastewater by constructed wetlands: Design, performance and mechanism
}

\author{
Yifei Li ${ }^{\text {a,* }}{ }^{\text {, Guibing Zhu }}{ }^{\text {b }}$, Wun Jern $\mathrm{Ng}^{\text {a,c }}$, Soon Keat Tan ${ }^{\mathrm{d}}$

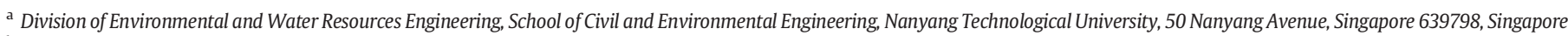 \\ b State Key Laboratory of Environmental Aquatic Quality, Research Center for Eco-Environmental Sciences, Chinese Academy of Sciences, 100085, PR China \\ ' Nanyang Environment and Water Research Institute, Nanyang Technological University, 1 Cleantech Loop, CleanTech One, Singapore 637141, Singapore

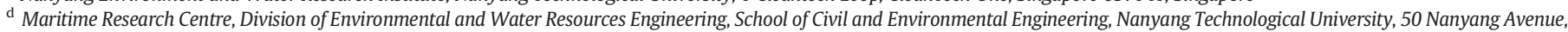 \\ Singapore 639798, Singapore
}

\section{H I G H L I G H T S}

- We review the application of constructed wetlands for pharmaceutical removal.

- We summarize and analyze the design parameters of the reported wetlands.

- Constructed wetlands show great potential for treatment of pharmaceuticals.

- Substrate, plants and microbes in wetlands account for the removal mechanisms.

- Relevant research is still limited and requires further work.

\section{A R T I C L E I N F O}

\section{Article history:}

Received 4 July 2013

Received in revised form 23 August 2013

Accepted 5 September 2013

Available online 1 October 2013

Editor: Damia Barcelo

\section{Keywords:}

Constructed wetland

Pharmaceutical

Emerging pollutant

Wastewater treatment

\begin{abstract}
A B S T R A C T
This paper presents a comprehensive review of the current state of research activities on the application of constructed wetlands for removing pharmaceutical contaminants from wastewater. The focus of the review was placed on the application of constructed wetlands as an alternative secondary wastewater treatment system or as a wastewater polishing treatment system. The design parameters of the reported constructed wetlands including the physical configuration, hydraulic mode, vegetation species, and targeting pharmaceuticals were summarized. The removal efficiencies of pharmaceuticals under different conditions in the wetlands were evaluated at the macroscopic level. In addition, the importance of the three main components of constructed wetlands (substrate, plants and microbes) for pharmaceutical removal was analyzed to elucidate the possible removal mechanisms involved. There is a general consensus among many researchers that constructed wetlands hold great potential of being used as an alternative secondary wastewater treatment system or as a wastewater polishing treatment system for the removal of pharmaceuticals, but relevant reported studies are scarce and are not conclusive in their findings. Current knowledge is limited on the removal efficiencies of pharmaceuticals in constructed wetlands, the removal mechanisms involved, the toxicity to constructed wetlands caused by pharmaceuticals, and the influences of certain important parameters (configuration design, hydraulic mode, temperature and seasonality, $\mathrm{pH}$, oxygen and redox potential, etc.). This review promotes further research on these issues to provide more and better convincing evidences for the function and performance of larger laboratory-scale, pilot-scale or full-scale constructed wetlands.
\end{abstract}

(c) 2013 Elsevier B.V. All rights reserved.

\section{Contents}

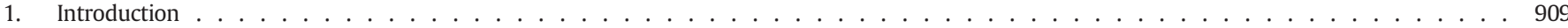

2. Design and performance of constructed wetlands for the removal of pharmaceuticals from wastewater . . . . . . . . . . . . . . . . . . . . 910

2.1. Reported pharmaceuticals and constructed wetlands . . . . . . . . . . . . . . . . . . . . . . . . . . . 910

2.2. Constructed wetlands as a secondary treatment for pharmaceutical removal . . . . . . . . . . . . . . . . . . . . . . . . . . 911

2.2.1. Design parameters of constructed wetlands . . . . . . . . . . . . . . . . . . . . . . . . . . . . . . . . . . . 911

2.2.2. Removal performance of pharmaceuticals in constructed wetlands . . . . . . . . . . . . . . . . . . . . . . . . . . 911

\footnotetext{
* Corresponding author. Tel.: +65 98320529.

E-mail addresses: yli19@e.ntu.edu.sg (Y. Li), gbzhu@rcees.ac.cn (G. Zhu), wjng@ntu.edu.sg (W.J. Ng), ctansk@ntu.edu.sg (S.K. Tan).
} 
2.3. Constructed wetlands as a polishing treatment for pharmaceutical removal . . . . . . . . . . . . . . . . . . . 917

2.3.1. Design parameters of constructed wetlands . . . . . . . . . . . . . . . . . . . . . . . . . . . 917

2.3.2. Removal performance of pharmaceuticals in constructed wetlands . . . . . . . . . . . . . . . . . . . . 921

3. Removal mechanisms related to the substrate, plants and microbes in constructed wetlands . . . . . . . . . . . . . . . . . . . . . . . . 922

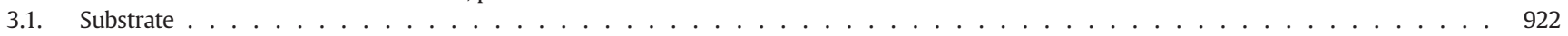

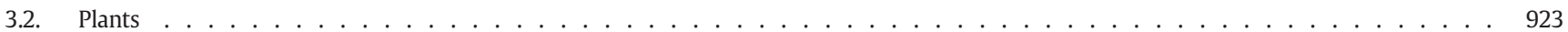

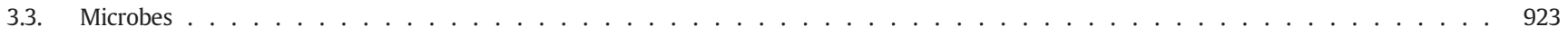

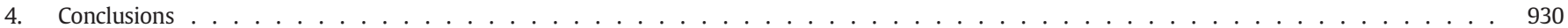

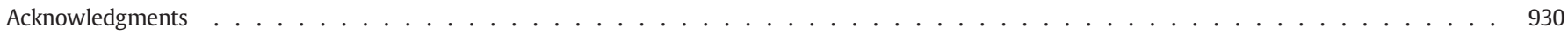

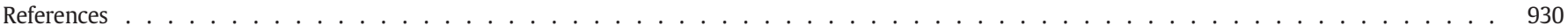

\section{Introduction}

Pharmaceuticals of different therapeutic classes are consumed in large number annually around the world to prevent, diagnose, mitigate or cure diseases in humans as well as animals (Jones et al., 2001, 2007; Uslu et al., 2013; Zhang et al., 2008). In recent years, with the advancement in analytical techniques of trace pharmaceutical residues, many studies have demonstrated the widespread occurrence of pharmaceuticals in water environment (Fent et al., 2006; Jiang et al., 2013; Jones et al., 2001; Uslu et al., 2013; Verlicchi et al., 2012; Yang et al., 2011). Increasing concern has been raised on this issue to investigate the source, behavior, fate, risk, and control of such emerging pollutants (Jiang et al., 2013; Pal et al., 2010; Richardson, 2007; J. Zhang et al., 2013).

The main route of pharmaceuticals brought into water environment is through the municipal wastewater. Many pharmaceuticals are not completely metabolized and ingested in the body of humans and animals, as a result, pharmaceutical metabolites, conjugates and their native forms are excreted with urine and feces into sewage system (Carballa et al., 2004; Jones et al., 2002; Zhang et al., 2008). In households, the unused and expired pharmaceuticals are usually disposed with normal household waste or discarded into sink or toilets (Zhang et al., 2008). Those pharmaceuticals flushed down the sink or toilets are introduced to the sewage system. Besides, to a minor but relevant extent, the wastewater from hospitals or pharmaceutical manufacturers also contributes to the total loads of pharmaceuticals in municipal wastewater (Fent et al., 2006; Jones et al., 2001; Santos et al., 2013; Saussereau et al., 2013). In the municipal wastewater treatment plants (WWTPs) where the conventional treatment technologies are not specially designed for elimination of pharmaceuticals, it is found that most pharmaceuticals cannot be readily and fully removed (Carballa et al., 2004; Jiang et al., 2013; Joss et al., 2006; Lindqvist et al., 2005; Paxéus, 2004; Petrie et al., 2013; Repice et al., 2013; Sim et al., 2010; Uslu et al., 2013; Vidal-Dorsch et al., 2012). Consequently, various kinds of pharmaceuticals and their metabolites are released into surface water, ground water, coastal water, and even drinking water via WWTPs' effluent (Jelic et al., 2011; Kim et al., 2007; Lindqvist et al., 2005; Uslu et al., 2013; VidalDorsch et al., 2012; Vieno et al., 2005).

In addition to the municipal wastewater as the major pathway of pharmaceutical compounds entering fresh or marine water environments, there exist some other direct pathways including the household wastewater from small communities, the runoff or wastewater effluent from animal feedlots, and the rural wastewater (Anderson et al., 2013; Carvalho et al., 2013; Focazio et al., 2008; Matamoros et al., 2009a).

Upon entering the water environment, the pharmaceutical compounds and their metabolites became potential risks to the health of aquatic life and human beings even at trace levels in the water environment (Carlsson et al., 2006; Corcoran et al., 2010; Farré et al., 2008; Fent et al., 2006; Gagné et al., 2006; Pomati et al., 2006; Zuccato et al., 2006). The adverse effects on aquatic communities include the feminization of male fish (Corcoran et al., 2010; Fent et al., 2006), impairment of renal, gill and liver in fish (Fent et al., 2006;
Gagné et al., 2006), development of pathogen resistance (Zuccato et al., 2006), and decrease in plankton diversity (Farré et al., 2008). In the case of the toxic impacts on human health, the degenerative and inflammatory reactions have been found in the liver of humans when exposing to diclofenac. (a type of analgesic/anti-inflammatory pharmaceutical) (Fent et al., 2006). In addition, the proliferation inhibition effects were observed for the growth of human embryonic cells under the injection of a pharmaceutical mixture (consisting of atenolol, carbamazepine, ciprofloxacin, furosemide, ibuprofen, sulfamethoxazole, etc.) (Pomati et al., 2006). At present, the information available on the ecotoxicology of pharmaceuticals is weak and the potential risks associated with the presence of pharmaceuticals in water environment are still under debate (Jones et al., 2004). Application of the precautionary principle, therefore, is required to give rise to more stringent controls on treatment of pharmaceuticals in wastewater.

In recent years, certain advanced technologies such as advanced oxidation processes (ozonation, photolysis and heterogeneous photolysis, Fenton and photo-Fenton, sonolysis, electrochemical oxidation, etc.), activated carbon adsorption, membrane separation, and membrane bioreactor have been investigated to assess their effectiveness for the removal of pharmaceuticals from wastewater (Klamerth et al., 2010; Martínez et al., in press; Mestre et al., 2009; MolinosSenante et al., 2013; Naddeo et al., 2009; Rossner et al., 2009; Singh et al., 2008; Sipma et al., 2010; Trinh et al., 2012). However, these advanced treatment processes are expensive making the large-scale application cost-prohibitive. Thus, selecting low-cost alternative technologies for pharmaceutical treatment is of great significance, especially in poor regions. For this purpose, constructed wetlands which are low-cost in construction, operation and maintenance are attracting great concern on their application for the removal of pharmaceutical contaminants from wastewater.

In the past decades, constructed wetlands have been demonstrated to be efficient for treatment of conventional pollutants in a variety of wastewaters such as domestic wastewater, agricultural wastewater, industrial effluent, mine drainage, leachate, contaminated ground water, and urban runoff (Choudhary et al., 2011; Cooper et al., 1996; Davies et al., 2008; García et al., 2010; Kadlec and Wallace, 2009; Stottmeister et al., 2003; Sundaravadivel and Vigneswaran, 2001; Vymazal, 2009; Vymazal et al., 1998). However, for the treatment of pharmaceutical contaminants in wastewater using constructed wetlands, it is really a fresh application field. The feasibility of constructed wetlands to eliminate pharmaceuticals in wastewater is requiring comprehensive understanding on the removal efficiencies, the removal mechanisms, the influences of design and environmental factors, and the toxicity risks. Hence, much more attention is needed to pay for these issues in future research studies.

The objective of this paper is to present the state of the research activities on the application of constructed wetlands for the removal of pharmaceutical contaminants from wastewater. The review focuses on the application of constructed wetlands as an alternative secondary wastewater treatment system or as a wastewater polishing treatment system. The design parameters of constructed wetlands 
were summarized to provide an understanding about the target pharmaceuticals, configuration, hydraulic mode and vegetation species of the reported constructed wetlands. The removal efficiencies of pharmaceuticals in constructed wetlands were also summarized in this paper in order to evaluate the performance of constructed wetlands in a macroscopic level. In addition, the possible removal mechanisms of pharmaceuticals related to the three important components of constructed wetlands (substrate, plants and microbes) were analyzed. The overall goal of this paper aims to be able to offer help for the further research in future.

\section{Design and performance of constructed wetlands for the removal of pharmaceuticals from wastewater}

\subsection{Reported pharmaceuticals and constructed wetlands}

115 pharmaceuticals grouped to 18 categories (according to their therapeutic classes) have been reported in 38 published papers in relation to their removal using constructed wetlands (including both the applications as a secondary wastewater treatment system and a polishing wastewater treatment system). The chemical structures and the physico-chemical properties of these pharmaceuticals can refer to the appendix form on the website of Maritime Research Centre in Nanyang Technological University (http://mrc.ntu.edu.sg/RD/Pages/ default.aspx). In the form on this website, the pharmaceutical compounds are presented in terms of their chemical formula, chemical structure, molecular weight (MW), dissociation constant $\left(\mathrm{pK}_{\mathrm{a}}\right)$, solubility $\left(\mathrm{S}_{\mathrm{w}}, 25^{\circ} \mathrm{C}\right)$, octanol-water partition coefficient as Log $\mathrm{K}_{\mathrm{ow}}$, and octanol-water distribution coefficient as $\log \mathrm{D}_{\mathrm{ow}}(\mathrm{pH}=7.4)$. These data were collected from relevant internet databases such as ChemIDplus Advanced, ChemSpider, DrugBank, Hazardous Substances Data Bank and Wikipedia. In addition, the number and details of the literature references reviewed are also indicated in the form. As deduced from the number of the references, the commonly investigated pharmaceuticals in constructed wetlands are diclofenac, ibuprofen, ketoprofen, naproxen, salicylic acid, sulfamethoxazole, triclosan, atenolol, clofibric acid, carbamazepine and caffeine.

The constructed wetlands used for the removal of pharmaceuticals from wastewater can be classified into surface free water constructed wetlands (SF-CWs), horizontal subsurface flow constructed wetlands (HSSF-CWs), vertical subsurface flow constructed wetlands (VSSFCWs) and hybrid constructed wetlands (hybrid CWs). The SF-CWs are composed of shallow channels or basins planted with vegetation (including rooted and floating plants) in which free wastewater flows at relatively shallow depth over the impermeable bottom liner or the packed substrate layer (Fig. 1a). In the HSSF-CW systems, wastewater is fed into the wetland at the inlet zone and flows horizontally through the substrate under the surface of wetland bed which is planted with vegetation (Fig. 1b). After the treatment of wetland, the wastewater effluent is collected at the outlet zone. For the VSSF-CW systems, wastewater is dosed onto the surface of wetland bed and then flows vertically from the planted layer down through the substrate

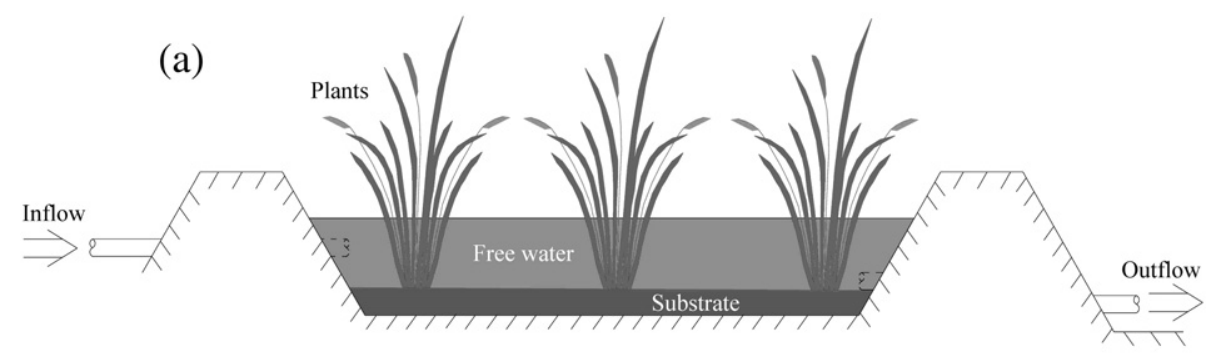

(b)
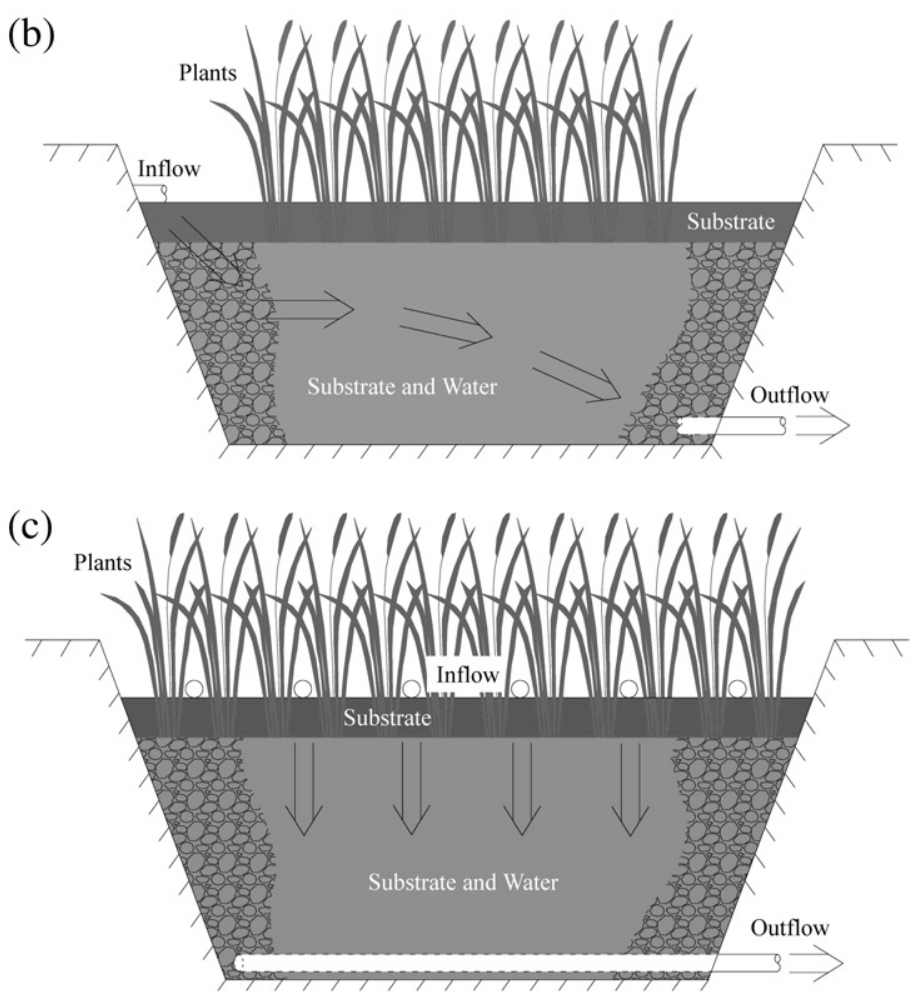

Fig. 1. Structures of constructed wetlands. (a) SF-CW; (b) HSSF-CW; (c) VSSF-CW 
until it reaches the outlet zone (Fig. 1c). The hybrid CW systems are the combination of two or more wetlands or the combination of wetlands with other pond systems such as lagoons and facultative ponds in parallel or in series. Such hybrid systems are normally laid out in two or three stages in order to realize the improvement of treatment efficiencies.

\subsection{Constructed wetlands as a secondary treatment for pharmaceutical removal}

Constructed wetlands working as an alternative secondary wastewater treatment system to remove pharmaceutical contaminants from wastewater are being paid with increasing concern in many countries such as Canada, China, Denmark, Italy, Portugal, Singapore, Spain and USA. The main design parameters of the constructed wetlands reported in research work are summarized in Table 1 . The removal efficiencies of the investigated pharmaceuticals by the secondary treatment of constructed wetlands are listed in Table 2 . The comparison between the removal efficiencies of constructed wetlands and that of conventional WWTPs is shown in Fig. 2. The removal performance of pharmaceuticals in different types of constructed wetlands is compared in Fig. 3.

\subsubsection{Design parameters of constructed wetlands}

As reported in Table 1 , the size of the investigated constructed wetlands was defined in this paper as microcosm-scale (surface area $<0.5 \mathrm{~m}^{2}$ ), mesocosm-scale (surface area in the range of 0.5 to $5 \mathrm{~m}^{2}$ ), pilot-scale (surface area in the range of 5 to $100 \mathrm{~m}^{2}$ ) and full-scale (surface area $>100 \mathrm{~m}^{2}$ ), of which the mesocosm-scale attracted the most attention. Various kinds of wastewater were fed into constructed wetlands, for example, urban wastewater from sewers or WWTPs, rural wastewater from rural communities, household wastewater from sparsely populated regions, swine wastewater from pig farms, and synthetic urban or agricultural wastewater. Except for the synthetic wastewater, most wastewater was preliminary or primary treated by screen, coarse solid tank, septic tank, homogenization tank, settling tank, aerobic reactor, or hydrolytic upflow sludge bed (HUSB). The target pharmaceutical contaminants in wastewater mainly covered analgesic/anti-inflammatory drugs, antibiotics, betablockers, diuretics, lipid regulators, psychiatric drugs, stimulants/ psychoactive drugs and veterinary drugs. The analgesic/anti-inflammatory drugs including diclofenac, ibuprofen, ketoprofen, naproxen and salicylic acid were of great concern.

Water depth is one of the important parameters for designing constructed wetlands. As shown in Table 1, the water depth in SFCWs was usually maintained no more than $30 \mathrm{~cm}$, but larger in the gravel SF-CW beds where $10-25 \mathrm{~cm}$ of free water was over the gravel layer (25-60 cm deep). In HSSF-CW systems, the water depth was commonly maintained $5 \mathrm{~cm}$ under the surface of substrate $(30-60 \mathrm{~cm}$ deep). However, Hussain et al. (2011) designed the water depth as $20 \mathrm{~cm}$ below the surface of substrate $(60 \mathrm{~cm}$ deep), and Ranieri et al. (2011) and Reyes-Contreras et al. (2011) maintained the water depth same as the level of substrate as $60-65 \mathrm{~cm}$ and $50 \mathrm{~cm}$, respectively. For the VSSF-CW systems, the water depth was controlled just below the surface of substrate in the study of Carvalho et al. (2013), but no more data could be obtained from a very small number of other literatures.

Different constructed wetland systems reported in the literatures were designed using different hydraulic retention time (HRT): 2 to 6 days for the SF-CWs, 2 to 4 days for the HSSF-CWs, 1 to 2 days for the VSSF-CWs, and 2 to 15 days for the hybrid CWs (Table 1). Under these different HRT conditions, both the batch and continuous operation modes were selected for research and both the planted wetland beds and the unplanted beds were investigated. In the planted wetland beds, various types of plants were applied, among which the most popular vegetations were Typha spp. and Phragmites spp. In some studies, the plant initial density was selected as 10 to 50 plants $\mathrm{m}^{-2}$

Regarding the substrate in constructed wetlands, gravel was the most common aggregate with design $\mathrm{D}_{60}$ of $3 \mathrm{~mm}$ to $8 \mathrm{~mm}, \mathrm{D}_{10}$ of $0.5 \mathrm{~mm}$ to $4 \mathrm{~mm}, \mathrm{C}_{\mathrm{u}}$ of 0.8 to 2 , and initial porosity of $40 \%-45 \%$ (Table 1). In addition, other substrates including stone, lava rock, volcanic rock, zeolite, soil/red soil, sandy soil and sandy clay loam were also found to be applied in some constructed wetlands. Hussain et al. $(2011,2012)$ used sandy soil or sandy clay loam soil in SF-CWs and sandy soil in HSSF-CWs. Ranieri et al. (2011) filled the HSSF-CWs with three layers including $10 \mathrm{~cm}$ layer of soil, $20 \mathrm{~cm}$ layer of stone, and 30 to $35 \mathrm{~cm}$ layer of gravel. Carvalho et al. (2013) built the VSSFCW bed with three layers consisting of $10 \mathrm{~cm}$ layer of roots' substrate, $2 \mathrm{~cm}$ layer of lava rock and $4 \mathrm{~cm}$ layer of gravel (from upper to lower). Liu et al. (2013a) constructed the VSSF-CW bed in a different way for layer distribution (from upper to lower) as: $20 \mathrm{~cm}$ layer of red soil, $30 \mathrm{~cm}$ layer of volcanic rock or zeolite and $10 \mathrm{~cm}$ layer of gravel.

\subsubsection{Removal performance of pharmaceuticals in constructed wetlands}

The removal efficiencies of pharmaceuticals by the secondary treatment of constructed wetlands are important criteria to evaluate the performance of constructed wetlands in a macroscopic level. In Table 2, the removal efficiencies of 36 investigated pharmaceuticals are listed for different constructed wetland systems including SFCW, HSSF-CW, VSSF-CW and hybrid CW systems, as well as the conventional WWTPs. The other investigated pharmaceuticals whose concentrations in wastewater were frequently below the limit of detection are not included in this Table. Considering the possible influences of plant, seasonality, configuration, operation mode and flow's saturation situation, some removal efficiencies of pharmaceuticals are presented in details, for example, efficiency (plant/unplanted), efficiency (winter/summer), efficiency (low water depth/high water depth), efficiency (continuous/batch) and efficiency (saturated flow/ unsaturated flow).

As shown in Table 2, the removal efficiencies of ciprofloxacin $\mathrm{HCl}$, oxytetracycline $\mathrm{HCl}$, nadolol, cotinine, enrofloxacin, monensin, narasin and salinomycin in conventional WWTPs have not been reported. Thus the comparison between the performance of constructed wetlands and conventional WWTPs for elimination of these pharmaceuticals is not premature at this stage. With reference to Table 2, it can be seen that the removal efficiencies of ciprofloxacin $\mathrm{HCl}$, oxytetracycline $\mathrm{HCl}$, nadolol, cotinine and enrofloxacin in constructed wetlands are high (mean removal efficiency $>70 \%$ ), while the removal efficiencies of monensin, narasin and salinomycin in constructed wetlands are relatively low in the range of $20 \%$ to $50 \%$. For the other pharmaceuticals listed in Table 2, most of their removal efficiencies in constructed wetlands appear to be as good as or even better than those in conventional WWTPs (graphically shown in Fig. 2). It can be deduced that constructed wetlands have good potential of being used as an alternative secondary wastewater treatment to remove pharmaceutical contaminants from wastewater.

According to the mean removal efficiencies of pharmaceuticals in constructed wetlands (calculated from Table 2), the pharmaceuticals can be categorized to the readily removed, moderately removed, low removed, and hardly removed. The pharmaceuticals that are readily removed by constructed wetlands (mean removal efficiency $>70 \%$ ) are acetaminophen, salicylic acid, sulfadiazine, sulfadimethoxine, sulfamethazine, sulfamethoxazole, sulfapyridine, trimethoprim, atenolol, metoprolol, furosemide, caffeine and tetracycline. The pharmaceuticals that are moderately removed by constructed wetlands (mean removal efficiency between $50 \%$ and $70 \%$ ) include ibuprofen, naproxen, doxycydine and gemfibrozil. The pharmaceuticals that show low removal efficiencies in constructed wetlands (mean removal efficiency between $20 \%$ and 50\%) are diclofenac, ketoprofen, amoxicillin, clarithromycin, triclosan, sotalol, clofibric acid and carbamazepine. 


\begin{tabular}{|c|c|c|c|c|c|c|c|}
\hline References & Size & Target pharmaceuticals & Influent water & $\begin{array}{l}\text { Preliminary/primary } \\
\text { treatment }\end{array}$ & Types of CW systems & $\begin{array}{l}\text { Configuration and hydraulic } \\
\text { conditions }\end{array}$ & Plants \\
\hline $\begin{array}{l}\text { Anderson et al. } \\
(2013)\end{array}$ & Full-scale & $\begin{array}{l}\text { Carbamazepine, } \\
\text { Gemfibrozil, } \\
\text { Sulfamethoxazole, } \\
\text { Sulfapyridine }\end{array}$ & $\begin{array}{l}\text { Rural wastewater } \\
\text { from the Rural } \\
\text { Municipality of St. } \\
\text { Clements near Grand } \\
\text { Marais, Manitoba, } \\
\text { Canada }\end{array}$ & Septic tank & $\begin{array}{l}\text { Hybrid lagoon and } \\
\text { SF-CW in series }\end{array}$ & $\begin{array}{l}\text { Two lagoons in series: } 134 \mathrm{~m} \text { long, } \\
134 \mathrm{~m} \text { wide and } 2.3 \mathrm{~m} \text { deep each; } \\
\text { water depth was } 1.5 \mathrm{~m} \text {; storage } \\
\text { volume was } 29,400 \mathrm{~m}^{3} \text { each. } \\
\text { Connecting channel: } 0.7 \mathrm{~km} \text { long } \\
\text { between lagoon and SF-CW. } \\
\mathrm{SF-CW} \text { : Five channel rows in a snaking } \\
\text { configuration; water depth was } 15 \text { to } \\
30 \mathrm{~cm} \text {. } \\
\text { Effluent discharge: Lake Winnipeg. }\end{array}$ & Typha spp. \\
\hline Ávila et al. (2013) & Mesocosm-scale & $\begin{array}{l}\text { Acetaminophen, } \\
\text { Diclofenac, } \\
\text { Ibuprofen }\end{array}$ & $\begin{array}{l}\text { Urban wastewater } \\
\text { from a municipal } \\
\text { sewer in Spain } \\
\left(84 \mathrm{~L} \mathrm{~d}^{-1} \text { and } 6 \mathrm{~g}\right. \\
\text { BOD m} \mathrm{m}^{-2} \mathrm{~d}^{-1} \text { for } \\
\text { each treatment line) }\end{array}$ & $\begin{array}{l}\text { Coarse screen followed } \\
\text { by a hydrolytic upflow } \\
\text { sludge bed (HUSB) or a } \\
\text { conventional settling } \\
\text { tank }\end{array}$ & Hybrid HSSF-CWs & $\begin{array}{l}\text { Three treatment lines: } 1 \text { ) Control line: } \\
\text { settler-wetland permanently saturat- } \\
\text { ed; } 2 \text { ) batch line: settler-wetland op- } \\
\text { erated with saturated/unsaturated } \\
\text { phases; } 3 \text { ) anaerobic line: HUSB- } \\
\text { wetland permanently saturated. } \\
\text { Layout of each treatment line: Two } \\
\text { small wetlands in parallel ( } 0.95 \mathrm{~m} \\
\text { long, } 0.7 \mathrm{~m} \text { wide and } 0.45 \mathrm{~m} \text { deep } \\
\text { each) and connected to a big wetland } \\
\text { in series ( } 1.5 \mathrm{~m} \text { long, } 1.1 \mathrm{~m} \text { wide and } \\
0.5 \mathrm{~m} \text { deep). } \\
\text { Water depth: } 25 \mathrm{~cm} \text {. } \\
\text { Substrate (gravel): } 30 \mathrm{~cm} \text { deep; } \\
\text { D60 = } 7.3 \mathrm{~mm} \text {; Cu }=0.83 \text {; initial po- } \\
\text { rosity was } 40 \% \text {. } \\
\text { HRT: } 2.5 \mathrm{~d} \text { per treatment line. }\end{array}$ & Phragmites australis \\
\hline Ávila et al. (2010) & Mesocosm-scale & $\begin{array}{l}\text { Diclofenac, } \\
\text { Ibuprofen, } \\
\text { Naproxen }\end{array}$ & $\begin{array}{l}\text { Urban wastewater } \\
\text { from a municipal } \\
\text { sewer in Spain } \\
\left(84 \mathrm{~L} \mathrm{~d}^{-1} \text { and } 4.7 \mathrm{~g}\right. \\
\left.\text { BOD m} \mathrm{D}^{-2} \mathrm{~d}^{-1}\right)\end{array}$ & $\begin{array}{l}\text { Coarse screen followed } \\
\text { by a HUSB }\end{array}$ & Hybrid HSSF-CWs & $\begin{array}{l}\text { Layout: Two small wetlands in parallel } \\
\left(0.65 \mathrm{~m}^{2} \text { each) and connected to a big }\right. \\
\text { wetland in series }\left(1.65 \mathrm{~m}^{2}\right) \text {. } \\
\text { Water depth: } 25 \mathrm{~cm} . \\
\text { Substrate (gravel): } 30 \mathrm{~cm} \text { deep; } \\
\text { D }_{60}=5 \mathrm{~mm} \text {; initial porosity was } \\
40 \% \text {. } \\
\text { HRT: } 3.5 \mathrm{~d} \text {. }\end{array}$ & Phragmites australis \\
\hline $\begin{array}{l}\text { Carvalho et al. } \\
\text { (2013) }\end{array}$ & Microcosm-scale & $\begin{array}{l}\text { Enrofloxacin, } \\
\text { Tetracycline }\end{array}$ & $\begin{array}{l}\text { Swine wastewater } \\
\text { from a pig farm in } \\
\text { Portugal }\end{array}$ & $--^{d}$ & VSSF-CW & $\begin{array}{l}\text { Sixteen microcosms: Seven were } \\
\text { planted and nine were left unplanted } \\
(0.4 \mathrm{~m} \text { long, } 0.3 \mathrm{~m} \text { wide and } 0.3 \mathrm{~m} \\
\text { deep for each container). } \\
\text { Substrate (from upper to lower): } \\
10 \mathrm{~cm} \text { layer of roots' bed substrate; } \\
2 \mathrm{~cm} \text { layer of lava rock; } 4 \mathrm{~cm} \text { layer of } \\
\text { gravel. } \\
\text { Water depth: Just below the substrate } \\
\text { surface (100\% flooding). } \\
\text { Loading mode: In batch. } \\
\text { Plant initial density: } 40 \text { plants per } \\
\text { microcosm. }\end{array}$ & Phragmites australis \\
\hline $\begin{array}{l}\text { Conkle et al. } \\
\text { (2008) }\end{array}$ & Full-scale & $\begin{array}{l}\text { Acetaminophen, } \\
\text { Atenolol, } \\
\text { Caffeine, } \\
\text { Carbamazepine, } \\
\text { Cotinine, } \\
\text { Fluoxetine, }\end{array}$ & $\begin{array}{l}\text { Urban wastewater } \\
\text { from Mandeville, } \\
\text { Louisiana, USA }\end{array}$ & - & $\begin{array}{l}\text { Hybrid aerated } \\
\text { lagoon and SF-CW in } \\
\text { series }\end{array}$ & $\begin{array}{l}\text { Three aerated lagoon in series: } 183 \mathrm{~m} \\
\text { long, } 61 \mathrm{~m} \text { wide and } 3 \mathrm{~m} \text { deep each; } \\
\text { HRT was } 9 \text { d each. } \\
\text { SF-CW: Water distribution was evenly } \\
\text { across } \\
\text { CW width by percolation through a }\end{array}$ & $\begin{array}{l}\text { Phragmites australis, } \\
\text { Hydrocottle spp. }\end{array}$ \\
\hline
\end{tabular}


Gemfibrozil,

Ibuprofen,

Metoprolo

Nadolol,

Naproxen,
Propranolol,

Sotalol,

Sulfamethoxazole,

Sulfapyridine

\begin{tabular}{|c|c|c|c|}
\hline $\begin{array}{l}\text { Hijosa-Valsero } \\
\text { et al. (2011a); } \\
\text { Hijosa-Valsero } \\
\text { et al. (2011c); } \\
\text { Hijosa-Valsero } \\
\text { et al. (2010b); } \\
\text { Reyes- } \\
\text { Contreras et al. } \\
\text { (2012) }\end{array}$ & Mesocosm-scale & $\begin{array}{l}\text { Hijosa-Valsero et al. } \\
\text { (2010b, 2011c) and } \\
\text { Reyes-Contreras et al. } \\
\text { (2012): } \\
\text { Caffeine, } \\
\text { Carbamazepine, } \\
\text { Diclofenac, } \\
\text { Ibuprofen, } \\
\text { Ketoprofen, } \\
\text { Naproxen, } \\
\text { Salicylic acid } \\
\text { Hijosa-Valsero et al. } \\
\text { (2011a): } \\
\text { Amoxicillin, } \\
\text { Ampicillin, } \\
\text { Clarithromycin, } \\
\text { Doxycycline, } \\
\text { Erythromycin, } \\
\text { Lincomycin, } \\
\text { Sulfamethoxazole, } \\
\text { Sulfadimethoxine, } \\
\text { Trimethoprim }\end{array}$ & $\begin{array}{l}\text { Urban wastewater } \\
\text { from the primary } \\
\text { clarifier of León } \\
\text { WWTP in Spain }\end{array}$ \\
\hline
\end{tabular}

crushed gravel bed; HRT was $1 \mathrm{~d}$ Effluent discharge: $60 \%$ was recycled, $40 \%$ was pumped into a ultra-violet irradiation channel for disinfection and then pumped into adjacent natural forested wetland for polishing before discharge into lake.

Container: $130 \mathrm{~cm}$ long, $80 \mathrm{~cm}$ wide and $50 \mathrm{~cm}$ deep; no gravel bed with floating macrophytes.

Water depth: $30 \mathrm{~cm}$.

Effluent pipe: At the top.

HRT: $2.1 \mathrm{~d}$.

Plant initial density: 50 plants $\mathrm{m}^{-2}$.
SF-CW

(with gravel)

SF-CW
(with gravel)

unplanted)

SF-CW

(soilless)

HSSF-CW

HSSF-CW

(unplanted)
Container:

Contain. $130 \mathrm{~cm}$ long, $80 \mathrm{~cm}$ wide

and $50 \mathrm{~cm}$ deep; $25 \mathrm{~cm}$ of free water

was over $25 \mathrm{~cm}$ layer of grave

$\left(D_{10}^{\mathrm{b}}=4 \mathrm{~mm}\right)$.

Effluent pipe: At the top.

HRT: $3.3 \mathrm{~d}$.

Plant initial density: 50 plants $\mathrm{m}^{-2}$.

Container: $130 \mathrm{~cm}$ long, $80 \mathrm{~cm}$ wide

and $50 \mathrm{~cm}$ deep; $25 \mathrm{~cm}$ of free water

was over $25 \mathrm{~cm}$ layer of gravel

$\left(D_{10}=4 \mathrm{~mm}\right)$.

Effluent pipe: At the bottom.

HRT: $5.1 \mathrm{~d}$.

Plant initial density: 50 plants $\mathrm{m}^{-2}$. Container: $130 \mathrm{~cm}$ long, $80 \mathrm{~cm}$ wide and $50 \mathrm{~cm}$ deep; $25 \mathrm{~cm}$ of free water was over $25 \mathrm{~cm}$ layer of grave

$\left(D_{10}=4 \mathrm{~mm}\right)$.

Effluent pipe: At the bottom.

HRT: $6.1 \mathrm{~d}$.

Container: $130 \mathrm{~cm}$ long, $80 \mathrm{~cm}$ wide and $50 \mathrm{~cm}$ deep; no gravel bed with floating macrophytes.

Water depth: $30 \mathrm{~cm}$.

Effluent pipe: At the top.

HRT: 2.9 d.

Plant initial density: 50 plants $\mathrm{m}^{-2}$. Container: $130 \mathrm{~cm}$ long, $80 \mathrm{~cm}$ wide and $50 \mathrm{~cm}$ deep.

Substrate (gravel): $45 \mathrm{~cm}$ deep;

$\mathrm{D}_{10}=4 \mathrm{~mm}$.

Water depth: $40 \mathrm{~cm}$.

Effluent pipe: At the bottom.

HRT: $2.5 \mathrm{~d}$.

Plant initial density: 50 plants $\mathrm{m}^{-2}$.

Container: $130 \mathrm{~cm}$ long, $80 \mathrm{~cm}$ wide

and $50 \mathrm{~cm}$ deep.

Substrate (gravel): $45 \mathrm{~cm}$ deep;

$\mathrm{D}_{10}=4 \mathrm{~mm}$.

Water depth: $40 \mathrm{~cm}$.

Effluent pipe: At the bottom.

HRT: 2.6 d.

Typha angustifolia

Typha angustifolia

Typha angustifolia

Phragmites australis

Unplanted 


\begin{tabular}{|c|c|c|c|c|c|c|c|}
\hline References & Size & Target pharmaceuticals & Influent water & $\begin{array}{l}\text { Preliminary/primary } \\
\text { treatment }\end{array}$ & Types of CW systems & $\begin{array}{l}\text { Configuration and hydraulic } \\
\text { conditions }\end{array}$ & Plants \\
\hline $\begin{array}{l}\text { Hijosa-Valsero } \\
\text { et al. (2011b); } \\
\text { Hijosa-Valsero } \\
\text { et al. (2011c) }\end{array}$ & & $\begin{array}{l}\text { Caffeine, } \\
\text { Carbamazepine, } \\
\text { Diclofenac, } \\
\text { Ibuprofen, } \\
\text { Ketoprofen, } \\
\text { Naproxen, } \\
\text { Salicylic acid }\end{array}$ & $\begin{array}{l}\text { Urban wastewater } \\
\text { from Barcelona } \\
\text { or León in Spain } \\
\left(84 \mathrm{Ld}^{-1}, 6 \mathrm{~g} \mathrm{BOD}_{5}\right. \\
\left.\mathrm{m}^{-2} \mathrm{~d}^{-1}\right)\end{array}$ & $\begin{array}{l}\text { Settling tank (ST) or } \\
\text { HUSB }\end{array}$ & & $\begin{array}{l}\text { Five treatment lines: Two CW tanks in } \\
\text { series in each line. } \\
\text { First CW tank: Two parallel compart- } \\
\text { ments, each } 93 \mathrm{~cm} \text { long, } 55 \mathrm{~cm} \text { wide } \\
\text { and } 45 \mathrm{~cm} \text { deep. } \\
\text { Second CW tank: } 134 \mathrm{~cm} \text { long, } \\
100 \mathrm{~cm} \text { wide and } 80 \mathrm{~cm} \text { deep. } \\
\text { Gravel depth: } 30 \mathrm{~cm} . \\
\text { Water depth: } 25 \mathrm{~cm} . \\
\text { HRT: } 3.5 \mathrm{~d} \text {. } \\
\text { Plant initial density: } 50 \text { plants } \mathrm{m}^{-2} \text {. }\end{array}$ & \\
\hline \multirow{3}{*}{$\begin{array}{l}\text { Hijosa-Valsero } \\
\text { et al. (2011c); } \\
\text { Hijosa-Valsero } \\
\text { et al. (2010a) }\end{array}$} & & $\begin{array}{l}\text { Naproxen, } \\
\text { Salicylic acid }\end{array}$ & & & & SF-CW & $\begin{array}{l}\text { Surface area: } 44 \mathrm{~m}^{2} \text {. } \\
\text { Gravel depth: } \\
30 \mathrm{~cm} . \\
\text { Gravel size: } 6- \\
8 \mathrm{~mm} . \\
\text { Water depth: } 40 \mathrm{~cm} \text {. } \\
\text { HRT: } 1.2 \mathrm{~d} \text {. }\end{array}$ \\
\hline & & & $\begin{array}{l}\text { Typha latifolia } \\
\text { HSSF-CW }\end{array}$ & $\begin{array}{l}\text { Surface area: } 585 \mathrm{~m}^{2} \text {. } \\
\text { Gravel depth: } 55 \mathrm{~cm} \text {. } \\
\text { Gravel size: } 6-8 \mathrm{~mm} \text {. } \\
\text { HRT: } 5.7 \mathrm{~d} .\end{array}$ & Salix atrocinerea & & \\
\hline & & & $\begin{array}{l}\text { Urban wastewater } \\
\text { from Bustillo de Cea } \\
\text { in Spain }\end{array}$ & $\begin{array}{l}\text { Bar screen and coarse } \\
\text { solid tank }\end{array}$ & $\begin{array}{l}\text { Hybrid facultative } \\
\text { pond (FP), SF-CW } \\
\text { and HSSF-CW in se- } \\
\text { ries }\end{array}$ & FP & $\begin{array}{l}\text { Surface area: } \\
230 \mathrm{~m}^{2} . \\
\text { Water depth: } 1.5- \\
2 \mathrm{~m} . \\
\text { HRT: } 4.21 \mathrm{~d} .\end{array}$ \\
\hline \multicolumn{8}{|r|}{ 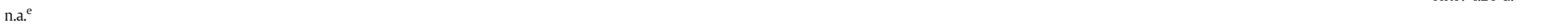 } \\
\hline SF-CW & $\begin{array}{l}\text { Surface area: } \\
210 \mathrm{~m}^{2} . \\
\text { HRT: } 3.53 \text { d. }\end{array}$ & Typha latifolia & & & & & \\
\hline HSSF-CW & $\begin{array}{l}\text { Surface area: } \\
362.5 \mathrm{~m}^{2} \text {. } \\
\text { HRT: } 3.16 \mathrm{~d} \text {. }\end{array}$ & Salix atrocinerea & & & & & \\
\hline $\begin{array}{l}\text { Hussain et al. } \\
\text { (2012) } \\
\text { Hussain et al. } \\
(2011)\end{array}$ & Mesocosm-scale & $\begin{array}{l}\text { Monensin, } \\
\text { Salinomycin, } \\
\text { Narasin }\end{array}$ & $\begin{array}{l}\text { Synthetic } \\
\text { agricultural } \\
\text { wastewater in } \\
\text { Canada }\left(1 \mathrm{~L} \mathrm{~min}^{-1}\right)\end{array}$ & - & $\begin{array}{l}\text { Hussain et al. (2012): } \\
\text { SF-CW } \\
\text { Hussain et al. (2011): } \\
\text { HSSF-CW }\end{array}$ & $\begin{array}{l}\text { Container: A high-density polyethyl- } \\
\text { ene half-pipe, } 6.1 \mathrm{~m} \text { long, } 1.5 \mathrm{~m} \text { wide } \\
\text { and } 0.75 \mathrm{~m} \text { deep; } 9.29 \mathrm{~m}^{2} ; 4.17 \mathrm{~m}^{3} \text {. } \\
\text { HRT: } 1) \text { SF-CW: } 4.1 \mathrm{~d} ; 2) \text { HSSF-CW: } \\
2.2 \mathrm{~d} \text {. } \\
\text { Water depth: } 1 \text { ) SF-CW: } 0.15 \mathrm{~m} \text { of } \\
\text { water was over } 0.6 \mathrm{~m} \text { of substrate; } 2 \text { ) } \\
\text { HSSF-CW: } 0.2 \mathrm{~m} \text { of water was below } \\
\text { substrate surface }(0.6 \mathrm{~m} \text { ) at the outlet. } \\
\text { Inlet zone: An inflow distribution } \\
\text { manifold and a transverse deep zone } \\
\text { without plants in } 2 \mathrm{~m} \text { length; } \\
\text { Treatment zone: A shallow zone } \\
\text { (4.1 m long) vegetated with alternate } \\
\text { bands of plants. } \\
\text { Outlet zone: A tipping bucket and a }\end{array}$ & $\begin{array}{l}\text { Phalaris arundinacea, } \\
\text { Typha latifolia }\end{array}$ \\
\hline
\end{tabular}




\begin{tabular}{|c|c|c|c|c|c|c|c|}
\hline Liu et al. (2013a) & Mesocosm-scale & $\begin{array}{l}\text { Ciprofloxacin } \mathrm{HCl}, \\
\text { Oxytetracycline } \mathrm{HCl}, \\
\text { Sulfamethazine }\end{array}$ & $\begin{array}{l}\text { Swine wastewater } \\
\text { from a pig farm in } \\
\text { Xiamen, China } \\
\left(3 \mathrm{~cm} \mathrm{~d}^{-1}\right)\end{array}$ & Aerobic reactor & VSSF-CW & $\begin{array}{l}\text { Toll. } \\
\text { Substrate (from upper to lower): } \\
20 \mathrm{~cm} \text { layer of red soil; } 30 \mathrm{~cm} \text { layer of } \\
\text { volcanic rock or zeolite; } 10 \mathrm{~cm} \text { layer of } \\
\text { gravel. } \\
\text { Plant initial density: } 15 \text { plants } \mathrm{m}^{-2} \text {. } \\
\text { HRT: } 30 \mathrm{~h} \text {. }\end{array}$ & Hybrid pennisetum \\
\hline $\begin{array}{l}\text { Matamoros et al. } \\
\text { (2009a) }\end{array}$ & $\begin{array}{l}\text { Full-scale } \\
\text { and } \\
\text { Pilot-scale }\end{array}$ & $\begin{array}{l}\text { Caffeine, } \\
\text { Carbamazepine, } \\
\text { Diclofenac, } \\
\text { Furosemide, } \\
\text { Ibuprofen, } \\
\text { Ketoprofen, } \\
\text { Naproxen, } \\
\text { Salicylic acid }\end{array}$ & $\begin{array}{l}\text { Household } \\
\text { wastewater from } \\
\text { sparsely populated } \\
\text { regions in the } \\
\text { eastern part of } \\
\text { Jutland peninsula of } \\
\text { Denmark }\end{array}$ & Settling tank & HSSF-CW, VSSF-CW & $\begin{array}{l}\text { Five HSSF-CWs' surface areas: } \\
500 \mathrm{~m}^{2} \text { (180 inhabitants); } \\
470 \mathrm{~m}^{2} \text { (80 inhabitants); } \\
940 \mathrm{~m}^{2} \text { (220 inhabitants); } \\
1800 \mathrm{~m}^{2} \text { (213 inhabitants); } \\
4000 \mathrm{~m}^{2} \text { (280 inhabitants). } \\
\text { Four VSSF-CWs' surface areas: } \\
16 \mathrm{~m}^{2} \text { (4 inhabitants); } \\
15 \mathrm{~m}^{2} \text { (2 inhabitants); } \\
15 \mathrm{~m}^{2} \text { (variable inhabitants); } \\
15 \mathrm{~m}^{2} \text { (4 inhabitants). }\end{array}$ & n.a. \\
\hline $\begin{array}{l}\text { Matamoros et al. } \\
\text { (2008a) }\end{array}$ & Mesocosm-scale & $\begin{array}{l}\text { Carbamazepine, } \\
\text { Clofibric acid, } \\
\text { Ibuprofen }\end{array}$ & $\begin{array}{l}\text { Synthetic urban } \\
\text { wastewater } \\
\text { (two organic carbon } \\
\text { sources: One was } \\
\text { starch; the other was } \\
\text { glucose) }\end{array}$ & - & HSSF-CW & $\begin{array}{l}\text { Container: } 0.93 \mathrm{~m} \text { long, } 0.59 \mathrm{~m} \text { wide } \\
\text { and } 0.52 \mathrm{~m} \text { deep. } \\
\text { Substrate (gravel): } 35 \mathrm{~cm} \text { deep; initial } \\
\text { porosity was } 40 \% ; \mathrm{D}_{60}=3.5 \mathrm{~mm} \text {; } \\
\mathrm{C}_{\mathrm{u}}=1.7 \text {. } \\
\text { Water depth: } 30 \mathrm{~cm} . \\
\text { Operating mode: } \text { In batch. }\end{array}$ & Phragmites australis \\
\hline $\begin{array}{l}\text { Matamoros et al. } \\
\text { (2007) }\end{array}$ & Pilot-scale & $\begin{array}{l}\text { Caffeine, } \\
\text { Carbamazepine, } \\
\text { Diclofenac, } \\
\text { Ibuprofen, } \\
\text { Naproxen, } \\
\text { Salicylic acid }\end{array}$ & $\begin{array}{l}\text { Urban wastewater } \\
\text { from vicinity of } \\
\text { Århus, Denmark } \\
\text { (loading rates: } 13, \\
30,70,160 \mathrm{~mm} \mathrm{~d}^{-1} \text { ) }\end{array}$ & $\begin{array}{l}\text { Three chamber settling } \\
\text { tank }\end{array}$ & VSSF-CW & $\begin{array}{l}\text { Surface area: } 5 \mathrm{~m}^{2} \text {. } \\
\text { Bottom gravel layer: } 8-16 \mathrm{~mm} \text { (size); } \\
0.2 \mathrm{~m} \text { deep. } \\
\text { Top gravel layer: } 0-4 \mathrm{~mm} \text { (size); } \\
0.8 \mathrm{~m} \text { deep; } \mathrm{D}_{10}=0.55 \mathrm{~mm} \text {; } \mathrm{D}_{60}= \\
3.1 \mathrm{~mm} \text {. } \\
\text { Water distribution: Four } 40 \mathrm{~mm} \\
\text { perforated pipes. }\end{array}$ & Phragmites australis \\
\hline $\begin{array}{l}\text { Matamoros and } \\
\text { Bayona (2006) } \\
\text { Matamoros } \\
\text { et al. (2005) }\end{array}$ & Pilot-scale & $\begin{array}{l}\text { Matamoros and Bayona } \\
\text { (2006): } \\
\text { Caffeine, } \\
\text { Diclofenac, } \\
\text { Ibuprofen, } \\
\text { Ketoprofen, } \\
\text { Naproxen, } \\
\text { Salicylic acid } \\
\text { Matamoros et al. (2005): } \\
\text { Carbamazepine, } \\
\text { Clofibric acid, } \\
\text { Ibuprofen }\end{array}$ & $\begin{array}{l}\text { Urban wastewater } \\
\text { from a housing } \\
\text { development in } \\
\text { Barcelona, Spain } \\
(200 \text { inhabitants, } \\
\left.36 \mathrm{~mm} \mathrm{~d}^{-1}\right)\end{array}$ & $\begin{array}{l}\text { Screen and Imhoff } \\
\text { settling tank }\end{array}$ & HSSF-CW & $\begin{array}{l}\text { Two parallel CW beds for comparison: } \\
\text { 1) Water depth was } 0.5 \mathrm{~m} \text {, aspect } \\
\text { ratio was } 2: 1 \text {, slope was } 1 \% ; 2 \text { ) water } \\
\text { depth was } 0.3 \mathrm{~m} \text {, aspect ratio was } \\
2.5: 1 \text {, slope was } 2.5 \% \text {. } \\
\text { Substrate (gravel): } \mathrm{D}_{60}=3.5 \mathrm{~mm} \text {; } \\
\mathrm{C}_{\mathrm{u}}=1.7 \text {; porosity was } 40 \% \text {; depth } \\
\text { was } 5 \mathrm{~cm} \text { above the water surface. } \\
\text { Surface area for each bed: } 54-56 \mathrm{~m}^{2} \text {. } \\
\text { Plant initial density: } 3 \text { plants } \mathrm{m}^{-2} \text {. }\end{array}$ & Phragmites australis \\
\hline $\begin{array}{l}\text { Ranieri et al. } \\
\text { (2011) }\end{array}$ & Pilot-scale & Paracetamol & $\begin{array}{l}\text { Wastewater near } \\
\text { Lecce, Italy }\end{array}$ & - & HSSF-CW & $\begin{array}{l}\text { Three HSSF-CWs: Phragmites bed, } \\
\text { Typha bed, and unplanted bed ( } 3 \mathrm{~m} \\
\text { wide, } 5 \mathrm{~m} \text { long, } 9.4 \mathrm{~m}^{3} \text { each). } \\
\text { Water depth: } 0.6 \mathrm{~m}-0.65 \mathrm{~m} \text {. } \\
\text { Substrate (upper to lower): } 0.1 \mathrm{~m} \text { of } \\
\text { soil layer; } 0.2 \mathrm{~m} \text { of stones layer; } 0.30- \\
0.35 \mathrm{~m} \text { of gravel layer. } \\
\text { Side banks: } 45^{\circ} \text { inclination. }\end{array}$ & $\begin{array}{l}\text { Phragmites australis, } \\
\text { Typha latifolia }\end{array}$ \\
\hline
\end{tabular}

sump.
Substrate: 1) In the six SF-CWs: three were filled with sandy clay loam soil; the other three were filled with sandy soil; 2) in the three HSSF-CWs: sandy

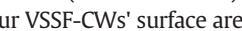

$\mathrm{m}^{2}$ (variable inhabitants);

was $40 \%$; $\mathrm{D}_{60}=3.5 \mathrm{~mm}$

2 m deep.

Two parallel CW beds for comparison:

Was $0.5 \mathrm{~m}$, aspect

gravel): $\mathrm{D}_{60}=3.5 \mathrm{~mm}$

1.7; porosity was $40 \%$; depth

Was 5 ch 
Table 1 (continued)

\begin{tabular}{|c|c|c|c|c|c|c|c|}
\hline References & Size & Target pharmaceuticals & Influent water & $\begin{array}{l}\text { Preliminary/primary } \\
\text { treatment }\end{array}$ & Types of CW systems & $\begin{array}{l}\text { Configuration and hydraulic } \\
\text { conditions }\end{array}$ & Plants \\
\hline $\begin{array}{l}\text { Reyes-Contreras } \\
\text { et al. (2011) }\end{array}$ & Pilot-scale & $\begin{array}{l}\text { Caffeine, } \\
\text { Carbamazepine, } \\
\text { Ibuprofen, } \\
\text { Ketoprofen, } \\
\text { Naproxen, } \\
\text { Salicylic acid, } \\
\text { Triclosan }\end{array}$ & $\begin{array}{l}\text { Wastewater from a } \\
\text { municipal } \\
\text { wastewater plant in } \\
\text { Santiago de } \\
\text { Compostela, } \\
\text { Galicia, Spain } \\
\left(17-20 \mathrm{~m}^{3} \mathrm{~d}^{-1}\right)\end{array}$ & $\begin{array}{l}\text { Upflow } \\
\text { anaerobic sludge blanket } \\
\text { (UASB) }\end{array}$ & $\begin{array}{l}\text { Hybrid SF-CW and } \\
\text { HSSF-CW in series }\end{array}$ & $\begin{array}{l}\text { Bottom slope: } 1 \% \text {. } \\
\text { Bottom liner: Bentonite liner (includ- } \\
\text { ing three layers: an upper geo-textile, } \\
\text { a lower geo-textile, and sodic pow- } \\
\text { dered bentonite). } \\
\text { Effluent discharge: Lagoon. } \\
\text { Surface area: } 75 \mathrm{~m}^{2} \text { each. } \\
\text { Plant density: } 2 \text { plants } \mathrm{m}^{-2} \text {. } \\
\text { Substrate (gravel): } 6-8 \mathrm{~mm} \text { (size); } \\
\text { initial porosity was } 0.45 ; 0.3 \mathrm{~m} \text { deep } \\
\text { for the SF-CW and } 0.5 \mathrm{~m} \text { deep for the } \\
\text { HSSF-CW. } \\
\text { Water depth: } 0.5 \mathrm{~m} \text {. } \\
\text { HRT: } 55 \mathrm{~h} \text {. }\end{array}$ & Juncus effusus \\
\hline Xian et al. (2010) & 列 & $\begin{array}{l}\text { Sulfadiazine, } \\
\text { Sulfamethazine, } \\
\text { Sulfamethoxazole }\end{array}$ & $\begin{array}{l}\text { Swine wastewater } \\
\text { from a concentrated } \\
\text { swine feeding plant } \\
\text { located in Nanjing, } \\
\text { China }\end{array}$ & - & SF-CW & $\begin{array}{l}\text { Container: } 0.5 \mathrm{~m} \text { long, } 0.4 \mathrm{~m} \text { wide and } \\
0.4 \mathrm{~m} \text { deep. } \\
\text { Working depth: } 0.3 \mathrm{~m} \text {. } \\
\text { Capacity volume: } 60 \mathrm{~L} \text {. } \\
\text { Foam plate for macrophytes floating: } \\
0.5 \mathrm{~m} \text { long, } 0.4 \mathrm{~m} \text { wide and } 0.015 \mathrm{~m} \\
\text { thick, with } 16 \text { rounded holes }(4 \times 4 \text {, } \\
2 \mathrm{~cm} \text { diameter). } \\
\text { Eight treatment systems (with } \\
\text { replications): Two Dryan beds, two } \\
\text { Waseyutaka beds, two Tachimasari } \\
\text { beds, and two unplanted beds. }\end{array}$ & $\begin{array}{l}\text { Ryegrass (Lolium): } \\
\text { Dryan, } \\
\text { Waseyutaka, } \\
\text { Tachimasari }\end{array}$ \\
\hline $\begin{array}{l}\text { Zhang et al. } \\
\text { (2012a) } \\
\text { Zhang et al. } \\
\text { (2012b) } \\
\text { Zhang et al. } \\
\text { (2011) }\end{array}$ & Mesocosm-scale & $\begin{array}{l}\text { Zhang et al. (2012a, } \\
\text { 2012b): } \\
\text { Caffeine, } \\
\text { Carbamazepine, } \\
\text { Clofibric acid, } \\
\text { Diclofenac, } \\
\text { Ibuprofen, } \\
\text { Ketoprofen, } \\
\text { Naproxen, } \\
\text { Salicylic acid } \\
\text { Zhang et al. (2011): } \\
\text { Carbamazepine, } \\
\text { Diclofenac, } \\
\text { Ibuprofen, } \\
\text { Naproxen }\end{array}$ & $\begin{array}{l}\text { Synthetic urban } \\
\text { wastewater in } \\
\text { Singapore }\end{array}$ & - & HSSF-CW & $\begin{array}{l}\text { Container: } 1.2 \mathrm{~m} \text { long, } 0.6 \mathrm{~m} \text { wide and } \\
0.6 \mathrm{~m} \text { deep. } \\
\text { Gravel depth: } 30 \mathrm{~cm} . \\
\text { Water depth: } 25 \mathrm{~cm} . \\
\text { HRT: } 2 \text { d or } 4 \text { d. } \\
\text { Plants initial density: } 14 \text { plants } \mathrm{m}^{-2} \text {. }\end{array}$ & Typha angustifolia \\
\hline
\end{tabular}


The pharmaceuticals that are hardly removed by constructed wetlands (average removal efficiency $<20 \%$ ) include ampicillin, erythromycin and lincomycin.

In Fig. 3, the removal efficiencies of different types of constructed wetland systems for the elimination of pharmaceuticals are compared. The VSSF-CW appears to be more efficient and reliable for the elimination of diclofenac, ibuprofen, naproxen, salicylic acid and caffeine than the CWs with other configurations. It can be attributed to their less sensitivity to overloading conditions, shorter hydraulic retention time (HRT) and better oxygenation in unsaturated flow (Matamoros et al., 2007; Matamoros et al., 2009a). However, only a very small number of studies have been conducted on VSSF-CW for the treatment of pharmaceutical contaminants. Thus the superiority of VSSF-CW for pharmaceutical removal over other types of constructed wetlands is still inconclusive.

The constructed wetland investigated most often is the HSSF-CW which has been applied separately or associated in hybrid CW system (together with lagoons, ponds, SF-CWs or other HSSF-CWs). As shown in Fig. 3, in comparison with the SF-CW, the HSSF-CW is more efficient for the removal of ketoprofen, naproxen, salicylic acid, doxycycline, sulfadimethoxine, trimethoprim, caffeine, monensin, narasin and salinomycin. Moreover, the HSSF-CW is comparably efficient for the removal of diclofenac, clarithromycin, sulfamethoxazole and carbamazepine, whereas less efficient for the removal of ibuprofen and amoxicillin. In addition, compared with the HSSF-CW for separate application, the hybrid CW system provides better removal efficiencies for acetaminophen, diclofenac, ibuprofen, naproxen and sulfamethoxazole, while lower removal efficiencies for ketoprofen, salicylic acid, carbamazepine and caffeine.

In summer, high temperature and strong sunlight irradiation were found to enhance the activities of plants and microorganisms in constructed wetlands, resulting in increased elimination of diclofenac, ibuprofen, ketoprofen, naproxen, salicylic acid, triclosan, carbamazepine and caffeine. The mean removal efficiencies of these pharmaceuticals were $23 \%, 53 \%, 30 \%, 61 \%, 82 \%, 48 \%, 25 \%$ and $90 \%$ during summer, in comparison to those of $20 \%, 38 \%, 22 \%, 39 \%, 56 \%, 7 \%, 16 \%$ and $53 \%$ correspondingly during winter (calculated from Table 2).

Hijosa-Valsero et al. (2010a) found that their most target pharmaceuticals, except for caffeine, ibuprofen and naproxen, were remarkably eliminated during the first stage regardless of the system design (a fullscale hybrid facultative pond, SF-CW and HSSF-CW system). Hussain et al. (2012) observed that the removal efficiencies of their target pharmaceuticals (monensin, salinomycin and narasin) in a mesocosm SF-CW system were significantly affected by the influent concentration levels. Matamoros and Bayona (2006) calculated the zero- or first-order areal rate constants for caffeine, ibuprofen and naproxen in a pilot-scale HSSF-CW system. They noted that the first-order kinetics fitted well with the decay of caffeine and ibuprofen. Zhang et al. (2012a) evaluated the first-order rate constants for caffeine, carbamazepine, clofibric acid, diclofenac, ibuprofen, ketoprofen, naproxen and salicylic acid in a mesocosm HSSF-CW system. They observed that the removal of caffeine, clofibric acid and ketoprofen followed the first-order decay kinetics. As stated above, within a certain period of residence time, the removal performance of certain pharmaceuticals in constructed wetlands would be related to the influent concentrations, resulting in variable removal efficiencies under different concentration levels.

Ávila et al. (2013) and Zhang et al. (2012b) observed the significant enhancement of the elimination of pharmaceuticals when feeding in batch mode due to the higher redox status caused by the alternating cycles of saturation and unsaturation. However, Hijosa-Valsero et al. (2011b) noted very little difference when using a batch flow or a continuous flow strategy. In addition, the hydraulic loading rate (HLR) or the hydraulic retention time (HRT) was suggested as another important hydraulic parameter influencing the removal efficiencies of pharmaceuticals in constructed wetlands. Ranieri et al. (2011) observed that the removal efficiency of paracetamol in a pilot-scale HSSF-CW system decreased as the HLR was increased. Zhang et al. (2012a) found that the removal efficiencies of all the target pharmaceuticals in a mesocosm HSSF-CW system were linearly proportional to the HRT. However, the difference was not obvious when the HRT was long enough for the treatment of pharmaceutical contaminants (Zhang et al., 2011).

\subsection{Constructed wetlands as a polishing treatment for pharmaceutical removal}

Constructed wetlands applied as a wastewater polishing treatment system to remove pharmaceutical contaminants from wastewater have been investigated in Denmark, Korea, Portugal, Sweden, Spain, UK and USA. In comparison to the publications on the application of constructed wetlands as an alternative secondary wastewater treatment system for the removal of pharmaceuticals, the number of the research studies on this application is relatively low. The design parameters of the reported constructed wetlands are summarized in Table 3. The removal efficiencies of the investigated pharmaceuticals in different types of constructed wetland systems are listed in Table 4.

\subsubsection{Design parameters of constructed wetlands}

As reported in Table 3, the size of the investigated constructed wetlands involved microcosm-scale (surface area $<0.5 \mathrm{~m}^{2}$ ), pilotscale (surface area in the range of 5 to $100 \mathrm{~m}^{2}$ ) and full-scale (surface area $>100 \mathrm{~m}^{2}$ ), of which the full-scale constructed wetlands attracted the most attention. The wastewater fed in the constructed wetlands included the secondary or tertiary effluent water from urban or rural WWTPs and the water from the rivers which received the effluent discharge of WWTPs. The target pharmaceutical contaminants in wastewater mainly covered analgesic/anti-inflammatory drugs, antiallergic drugs, antibiotics, antidiabetics, antidysenterics, antifungals, antihypertensives, anti-senile dementia drugs, barbiturates, beta-agonists, beta-blockers, diuretics, hormone inhibitors, lipid regulators, psychiatric drugs, receptor antagonists, stimulants/psychoactive drugs and veterinary drugs. Among these pharmaceuticals, diclofenac, ibuprofen, ketoprofen, naproxen, sulfamethoxazole, triclosan, atenolol, clofibric acid, carbamazepine and caffeine were the commonly investigated pharmaceuticals.

The constructed wetlands selected for wastewater polishing treatment (Table 3 ) included SF-CWs, HSSF-CWs, VSSF-CWs and hybrid CWs, of which the most popular wetlands were the SF-CWs and the hybrid SF-CWs. The SF-CWs reported by Llorens et al. (2009); Matamoros et al. (2012a); Matamoros et al. (2008b); and Zarate et al. (2012) were designed with surface area of 0.2 to 100 ha. and water depth of 0.05 to $2 \mathrm{~m}$. The target wastewater was retained in these SF-CWs for 3 to 30 days and was finally discharged into the surrounding lake or river. In the hybrid SF-CW system used by Breitholtz et al. (2012); Gross et al. (2004); Lee et al. (2011); and Park et al. (2009), two or more stages of wetland basins were laid out in parallel or in series covering a planar area of $0.36-130$ ha. with water depth in the range of 0.1 to $1 \mathrm{~m}$. The wastewater was typically retained in the system for $6 \mathrm{~h}$ to 14 days and was finally discharged into the surrounding lake, river or sea. Moreover, SF-CWs could also include ponds to form a hybrid system for wastewater polishing treatment. Matamoros and Salvadó (2012) conducted a study on such kind of hybrid system which consisted of two parallel ponds ( 2 ha. area and $1 \mathrm{~m}$ water depth for each) followed by three parallel deep SF-CWs ( 0.8 ha. area and $0.5 \mathrm{~m}$ water depth for each) and a large shallow SF-CW (4.5 ha. area and $0.2 \mathrm{~m}$ water depth). The wastewater was retained in the ponds and the SF-CWs (including both the deep and shallow SF-CWs) for 4 days and 8.5 days, respectively, and was finally pumped to an 18 ha. artificial pond in order to maintain the flooding of the marshlands.

For the HSSF-CWs, Dordio et al. (2010); Dordio et al. (2009a); and Verlicchi et al. (2013) evaluated the performance of HSSF-CWs for the removal of pharmaceuticals from the secondary effluent of 
Table 2

Removal efficiencies of pharmaceuticals in constructed wetlands applied as an alternative secondary wastewater treatment system.

\begin{tabular}{|c|c|c|c|c|c|}
\hline \multirow[t]{2}{*}{ Pharmaceutical compounds } & \multicolumn{5}{|l|}{ Removal efficiencies (\%) } \\
\hline & SF-CW & HSSF-CW & VSSF-CW & Hybrid CW system & Conventional WWTPs \\
\hline \multicolumn{6}{|l|}{ Analgesic/anti-inflammatory } \\
\hline Acetaminophen (paracetamol) & & 47 to $>99(14)$ & & $>99(19) ;>95(20)$ & $\begin{array}{l}>90(39) ; 98 \text { to }>99(40) \\
70-90(41) ; 96(43) ; \\
72-97(44) ;>99(46)\end{array}$ \\
\hline Diclofenac & $\begin{array}{l}20-40 \text { (winter, } 1,2,3) \\
0-50 \text { (summer, } 1,2,3 \text { ) }\end{array}$ & $\begin{array}{l}\text { 10-15 (winter, 1, 2, 3); } \\
\text { 0-35 (summer, 1, 2, 3); } 21 \text { (7); } \\
\text { 0-11 (0.5 m depth, 8); } \\
\text { 0-45 (0.3 m depth, 8); } \\
\text { 41-44 (continuous, planted, 9, 10); } \\
\text { 48-55 (continuous, planted, 11); } \\
\text { 24-32 (continuous, unplanted, 9); } \\
\text { 41-47 (continuous, unplanted, 11); } \\
\text { 49-55 (batch, planted, 10) }\end{array}$ & $\begin{array}{l}73 \text { (unsaturated flow } 16 \text { ); } \\
53 \text { (saturated flow } 16 \text { ) }\end{array}$ & $\begin{array}{l}\text { 32-70 (20); } \\
\text { 0-22 (winter, 21); } \\
\text { 0-71 (summer, 21); } \\
78-87 \text { (22) }\end{array}$ & $\begin{array}{l}17(24,25) ; 69(27) ; \\
22(29) ; 7-63(30) ; \\
0-70(31) ; 33(35) ; \\
49(38) ; 17-33(41) ; \\
39-75(42) ; 35(43) ; \\
0-80(44) ; 31(46) ; \\
42(47)\end{array}$ \\
\hline Ibuprofen & $\begin{array}{l}\text { 45-75 (winter, 1, 2, 3); } \\
\text { 55-95 (summer, 1, 2, 3) }\end{array}$ & $\begin{array}{l}\text { 25-40 (winter, 1, 2, 3); } \\
\text { 5-55 (summer, 1, 2, 3); } 65 \text { (7); } \\
\text { 17-52 (0.5 m depth, 8); } \\
48 \text { (0.5 m depth, 13); } \\
\text { 62-80 (0.3 m depth, 8); } \\
\text { 81 (0.3 m depth, 13); } \\
\text { 68-72 (continuous, planted, 9, 10); } \\
\text { 71-80 (continuous, planted, 11); } \\
\text { 52-61 (continuous, unplanted, 9); } \\
\text { 57-60 (continuous, unplanted, 11); } \\
\text { 72-83 (batch, planted, 10); 52 (12) }\end{array}$ & $\begin{array}{l}99 \text { (unsaturated flow 16); } \\
55 \text { (saturated flow 16); } \\
89 \text { (7) }\end{array}$ & $\begin{array}{l}>99(19) ; 52-85 \text { (20); } \\
0-47 \text { (winter, 21); } \\
35-99 \text { (summer, 21); } \\
\text { 42-89 (22); 98-99 (23); } \\
3 \text { (winter)-42 (summer) (48) }\end{array}$ & $\begin{array}{l}60-70(26) ; \\
90(22,27,28) ; \\
96(29,35) ; 99(30) ; \\
87(38) ; 87-93(41) ; \\
100(42) ; 88(43) ; \\
56-85(44) ; 80-87(45) ; \\
94 \text { (46); } 98(47)\end{array}$ \\
\hline Ketoprofen & $35-50$ (winter, 1, 2, 3) & $\begin{array}{l}\text { 10-20 (winter, 1, 2, 3); } 90 \text { (7); } \\
\text { n.r. }{ }^{\text {a }} \text { (0.5 m depth, 8); } \\
\text { 0-69 (0.3 m depth, 8); } \\
\text { 91-96 (continuous, planted, 9); } \\
\text { 91-92 (continuous, planted, 10); } \\
\text { 90-94 (continuous, unplanted, 9); } \\
\text { 94-95 (planted, batch, 10) }\end{array}$ & & $\begin{array}{l}\text { 4-27 (winter, 21); } \\
0-37 \text { (summer, 21); } \\
77-81 \text { (22); } \\
5 \text { (winter)-42 (summer) (48) }\end{array}$ & $\begin{array}{l}23(25) ; 30(28) ; 65(29) ; \\
68 \text { (38); } 70-84(41) ; \\
83-89 \text { (42); } 72-97 \text { ( } 44): 52-72(45) ; 85(46) ; 55(47)\end{array}$ \\
\hline Naproxen & $\begin{array}{l}\text { 40-65 (winter, 1, 2, 3); } \\
25-75 \text { (summer, 1, 2, 3) }\end{array}$ & $\begin{array}{l}\text { 25-45 (winter, 1, 2, 3) } \\
\text { 55-85 (summer, 1, 2, 3); } 45 \text { (7); } \\
\text { 0-47 (0.5 m depth, 8); } \\
\text { 80-90 (0.3 m depth, 8); } \\
\text { 81-93 (continuous, planted, 9, 10); } \\
\text { 83-91 (continuous, planted, 11); } \\
\text { 52-78 (continuous, unplanted, 9); } \\
\text { 50-52 (continuous, unplanted, 11) } \\
\text { 82-91(batch, planted, 10) }\end{array}$ & $\begin{array}{l}89 \text { (unsaturated flow 16); } \\
62 \text { (saturated flow 16); } \\
92 \text { (7) }\end{array}$ & $\begin{array}{l}\text { 98(19); } \\
0-41 \text { (winter, 21); } \\
\text { 60-95 (summer, 21); } \\
\text { 73-82 (22); } 99 \text { (23); } \\
30 \text { (winter)-53 (summer) (48) }\end{array}$ & $\begin{array}{l}82(24,25) ; 40-55(6) ; \\
66(27) ; 83(28) ; \\
89 \text { (22); } 93(29,38) ; \\
91-95(42) ; 67(43) ; \\
72-97(44) ; 43-71(45) ; \\
86 \text { (46); } 64(47)\end{array}$ \\
\hline
\end{tabular}


(2)

98 (unsaturated flow 16 ): 85 (saturated flow 16 ):

$87(7)$

86-90 (continuous, planted, 9);

88-89 (continuous, planted, 10):

88 (continuous, unplanted, 9);

93-94 (batch, planted, 10)

Antibiotics

Amoxicillin

Ampicillin

Ciprofloxacin $\mathrm{HC}$

Clarithromycin

Doxycycline

Erythromycin

Lincomycin

Oxytetracycline $\mathrm{HC}$

Sulfadiazine

Sulfadimethoxine

Sulfamethazine

Sulfamethoxazole

\section{Sulfapyridine}

Triclosan

Trimethoprim

$$
\begin{aligned}
& 27-45(4) \\
& 0-32(4)
\end{aligned}
$$

$11-50(4)$

$47-75(4)$

n.r. (4)

n.r. (4)

98-99 (5)

67-99 (4)

$99(5)$

59-80 (4); 89-92 (5)
73-87 (4)

95-99 (4)

$6-21(4)$

.r. (4)

$71-79(4)$

$0-64(4)$

n.r. (4)

(4)

91 (19)

76 (19)

7 (winter)-48 (summer) (48)

93 (19)

92 (19)

77 (19)

$30(19)$
80 (22); 88 (25); 95 (28);

$99(32,38) ; 94$ (43);

99 (46)

7 (22):

53 (winter)-70 (summer) (48)

$35(4)$

7 (4); n.r. (44)

\section{$18(4)$
$61(4)$}

n.r. (4); 25 (35);

-17 to $50(40)$

24 (4); 64-80 (40)

20-82 (44)

53 (4); 20-82 (44)

7-17 (41); 20-82 (44)

2-66 (30);24 (35):

(4)

$0-88$ (44); 83 (46)

$95(28)$

58 (29); 91-93 (42)

$14(4) ; 49(29) ; 69$ (35);

74 (38); $67-80$ (40);

67-89 (41); 22-56 (44);

$70(46)$

50-80 (29); 84 (35):

$73-79$ (36); 58 (37); 83-87(41); 71-77 (44)

83 (27); 30-65 (29);

65 (35); 29-31 (36);

17 (37); 3-67 (44)

48 (35); $26-27(36) ;$

$77(46)$ 
Table 2 (continued)

\begin{tabular}{|c|c|c|c|c|c|}
\hline \multirow[t]{2}{*}{ Pharmaceutical compounds } & \multicolumn{5}{|l|}{ Removal efficiencies (\%) } \\
\hline & SF-CW & HSSF-CW & VSSF-CW & Hybrid CW system & Conventional WWTPs \\
\hline \multicolumn{6}{|l|}{ Lipid regulators } \\
\hline Clofibric acid & & $\begin{array}{l}\text { 34-39 (continuous, planted, 9, 10); } \\
\text { 33-34 (continuous, unplanted, 9); } \\
\text { 33-34 (batch, planted, } 10) ; \\
\text { n.r. ( } 0.5 \text { m depth, } 13) ; \\
\text { n.r. }(0.3 \text { m depth, } 13 \text { ) }\end{array}$ & & & $\begin{array}{l}51(27) ; 52(35) ; \\
49(38) ; 0-81(44) ; \\
50(47)\end{array}$ \\
\hline Gemfibrozil & & & & $61(19)$ & $\begin{array}{l}69 \text { (27); } 75(29) ; 65 \text { (38); } \\
56 \text { (43); 0-81 (44) }\end{array}$ \\
\hline \multicolumn{6}{|l|}{ Psychiatric drugs } \\
\hline Carbamazepine & $\begin{array}{l}0-35 \text { (winter, 1, 2, 3); } \\
10-50 \text { (summer, 1, 2, 3) }\end{array}$ & $\begin{array}{l}\text { 20-25 (winter, 1, 2, 3,) } \\
\text { 0-40 (summer, 1, 2, 3); } 38 \text { (7); } \\
\text { 27-28 (continuous, planted, 9, 10, 11); } \\
\text { 24-26 (continuous, unplanted, 9); } \\
\text { 28-29 (continuous, unplanted, 11); } \\
\text { 27-28 (batch, planted, 10); 5 (12); } \\
26 \text { (0.5 m depth, 13); } \\
16 \text { (0.3 m depth, 13) }\end{array}$ & $\begin{array}{l}26 \text { (unsaturated flow } 16) ; \\
20 \text { (saturated flow } 16 \text { ) }\end{array}$ & $\begin{array}{l}0-9 \text { (winter, 21); } \\
0-58 \text { (summer, 21); } \\
5 \text { (winter)-18 (summer) (48) }\end{array}$ & $\begin{array}{l}7(27) ; 8(24,25,33) ; \\
15(22) ; 30(29,38) ; \\
-43 \text { to } 14(30) ; 0-30(31) ; \\
-13(43) ; 0-40(44) ; \\
7-11(45) ; 13(46)\end{array}$ \\
\hline \multicolumn{6}{|l|}{ Stimulants/psychoactive drugs } \\
\hline Caffeine & $\begin{array}{l}\text { 25-60 (winter, 1, 2, 3); } \\
\text { 80-99 (summer, 1, 2, 3) }\end{array}$ & $\begin{array}{l}\text { 60-65 (winter, 1, 2, 3); } \\
\text { 99 (summer, 1, 2, 3); 97 (7); } \\
\text { 85-94 (0.5 m depth, 8); } \\
\text { 94-99 (3 m depth, 8); } \\
\text { 84-90 (continuous, planted, 9, 10); } \\
\text { 82-84 (continuous, unplanted, 9); } \\
\text { 86-93 (batch, planted, 10) }\end{array}$ & $\begin{array}{l}99 \text { (unsaturated flow 16); } \\
82 \text { (saturated flow 16); } \\
99 \text { ( } 7 \text { ) }\end{array}$ & $\begin{array}{l}\text { >99 (19); } \\
\text { 14-84 (winter, 21); } \\
\text { 98-99 (summer, 21); } \\
\text { 95-96 (22); } \\
\text { 55 (summer)-60 (winter) (48) }\end{array}$ & $\begin{array}{l}86 \text { (22); } 93(28) ; \\
94 \text { (29); } 99(24,25,33) ; \\
\text { >99 (34); 87-93 (40); } \\
\text { 83-90 (41); } 89(43) ; \\
85-100 \text { (44); 44-75 (45) }\end{array}$ \\
\hline Cotinine & & & & $99(19)$ & \\
\hline \multicolumn{6}{|l|}{ Veterinary drugs } \\
\hline Enrofloxacin & & & $94(18)$ & & \\
\hline Monensin & $27-34(6)$ & $37-43(15)$ & & & \\
\hline Narasin & $29-36(6)$ & $42-57(15)$ & & & \\
\hline Salinomycin & $30-32(6)$ & $41-56(15)$ & & & \\
\hline Tetracycline & & & $98(18)$ & & $66-90(44)$ \\
\hline
\end{tabular}

Notes: a n.r.: not removed.

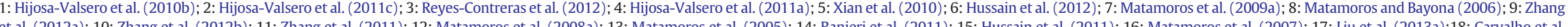

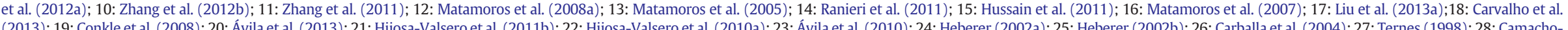

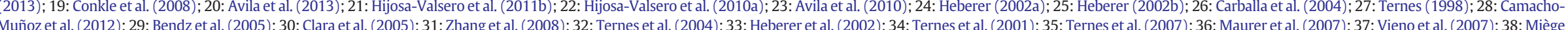

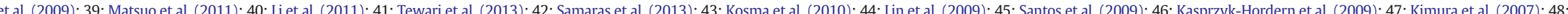

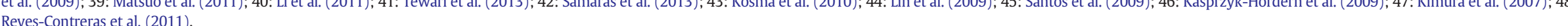




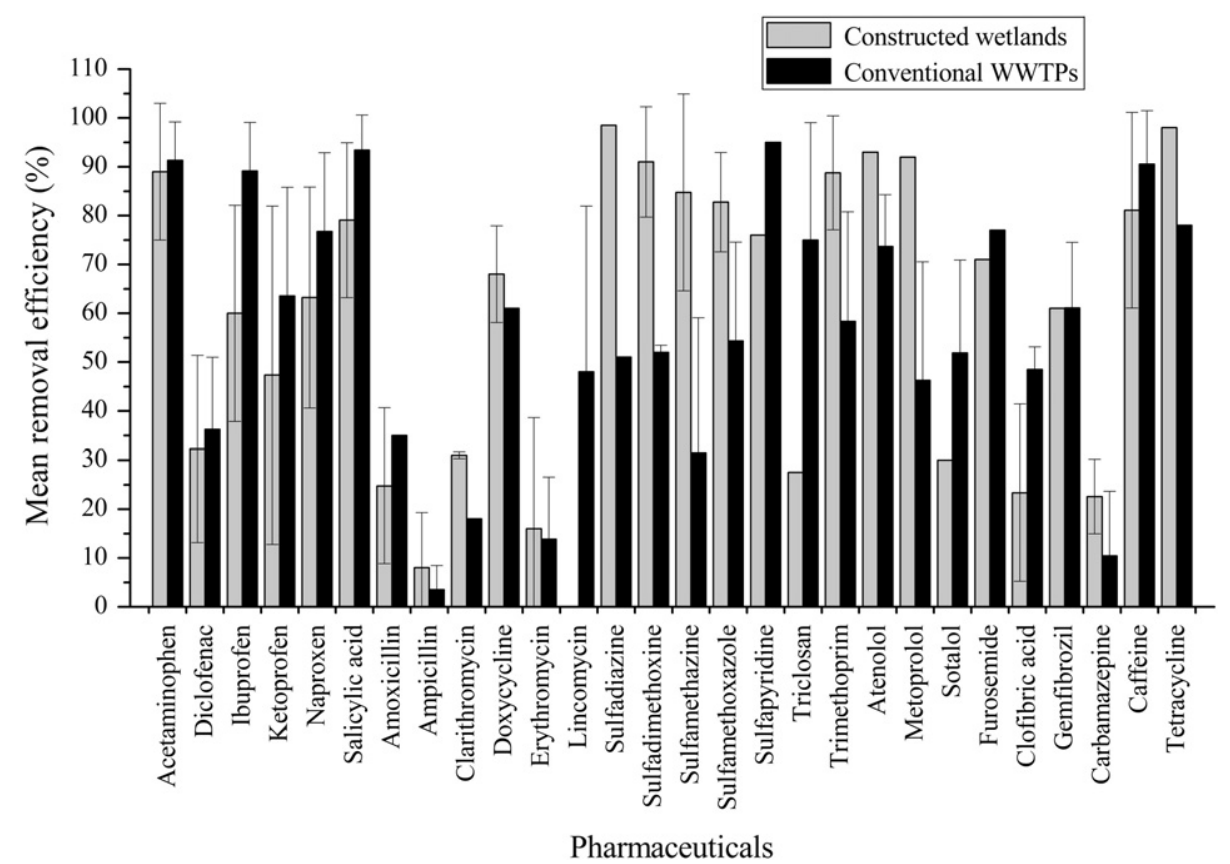

Fig. 2. Comparison between the mean removal efficiencies of pharmaceuticals in CWs and conventional WWTPs.

WWTPs. Their investigated HSSF-CWs were designed with a surface area of $0.3 \mathrm{~m}^{2}$ and $28 \mathrm{~m}^{2}$, respectively, and with a water depth of $0.3 \mathrm{~m}$ and $2.2 \mathrm{~m}$, respectively. Regarding the VSSF-CW, Reif et al. (2011) studied a pilot-scale VSSF-CW in batch operating mode for purifying the wastewater effluent from a WWTP in USA. In their research work, they designed the substrate layers of the wetland from top to bottom as: $0.15 \mathrm{~m}$ of filter grade sand, $0.15 \mathrm{~m}$ of $10 \mathrm{~mm}$ pea gravel, $0.05 \mathrm{~m}$ of $20 \mathrm{~mm}$ gravel and $0.6 \mathrm{~m}$ of $40-50 \mathrm{~mm}$ gravel.

In all the reported constructed wetlands, there were various types of vegetations, among which the most popular plants were Typha spp. and Phragmites spp.
2.3.2. Removal performance of pharmaceuticals in constructed wetlands As shown in Table 4, the data on the removal efficiencies of the investigated pharmaceuticals for each kind of constructed wetland are very limited. Thus the comparison among the different types of constructed wetlands is not feasible to date. For the commonly investigated pharmaceuticals, except for the low or inconsistent removal efficiencies obtained by Matamoros et al. (2012a); Breitholtz et al. (2012); and Verlicchi et al. (2013), the readily removed pharmaceuticals by constructed wetlands (mean removal efficiency $>70 \%$ ) are diclofenac, ibuprofen, ketoprofen, triclosan and atenolol. The moderately removed pharmaceuticals by constructed wetlands (mean removal efficiency

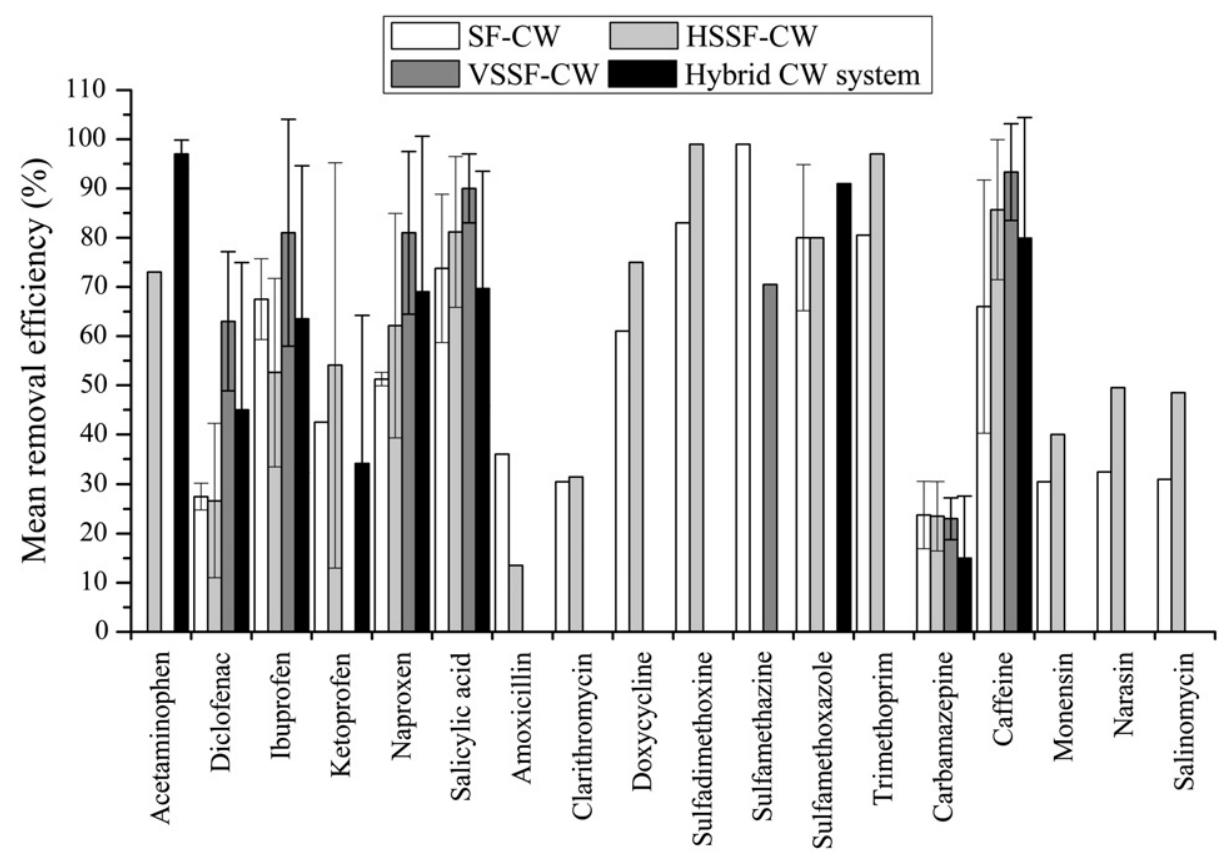

Pharmaceuticals

Fig. 3. Mean removal efficiencies of pharmaceuticals in different CW systems. 
between $50 \%$ and $70 \%$ ) include naproxen and caffeine; while the pharmaceuticals with low removal efficiencies in constructed wetlands (mean removal efficiency between $20 \%$ and 50\%) are sulfamethoxazole, clofibric acid and carbamazepine. Breitholtz et al. (2012) investigated four full-scale hybrid SF-CWs systems with different design configurations to remove pharmaceutical compounds from four sewage treatment plants. They detected that some pharmaceuticals were at higher levels in effluent water compared to that in the influent water (shown as negative removal efficiencies in Table 4). This phenomenon could partly be attributed to the numerical variations caused by the occurrence of pharmaceuticals close to the detection limit and the daily concentration fluctuations of pharmaceuticals induced by intermittent usage. In addition, it could also be possible because many pharmaceuticals were metabolized as glucuronides or other conjugated metabolites and then after a period of time were converted to the parent compounds by enzymatic processes.

Dordio et al. (2009a, 2010) found that the removal kinetics of atenolol, carbamazepine, clofibric acid and ibuprofen was a fast initial step in which more than half of the initial pharmaceuticals were removed within the first $6 \mathrm{~h}$ and followed by a first-order kinetics during the next period of 6-96 h. Matamoros et al. (2008b) observed that higher removal efficiencies of their target pharmaceuticals were obtained during the first part of system compared to the latter part of system, showing a spatial trend of the removal efficiencies. This phenomenon demonstrated that the first-order removal kinetics was appropriate for the removal of carbamazepine, diclofenac, flunixin, ibuprofen and ketoprofen. As stated above, within a certain period of residence time, the removal efficiencies of certain pharmaceuticals in constructed wetlands would be related to their influent concentrations.

\section{Removal mechanisms related to the substrate, plants and microbes in constructed wetlands}

\subsection{Substrate}

Substrate (also known as support matrix) is an important component in constructed wetlands, especially in subsurface flow constructed wetlands. The substrate in constructed wetlands not only provides support for the growth of plants and microorganisms, but also interacts directly with contaminants through sorption processes. Sorption of pollutants onto the surface of substrate involves different mechanisms such as hydrophobic partitioning, van der Waals interaction, electrostatic interaction, ion exchange, and surface complexation (Dordio and Carvalho, 2013; Haberl et al., 2003; Pei et al., 2012; Reddy and DeLaune, 2008; Tolls, 2001). Non-polar organic pollutants can be preferentially adsorbed to the substrate materials that are especially rich in organic matter such as soil, compost and agricultural wastes via hydrophobic process. Polar or ionic pollutants are dominantly adsorbed to the substrate materials (e.g. some kinds of clay) by electrostatic interactions or ionic exchange.

According to Table 1 and Table 3, the most frequently used substrate for pharmaceutical removal in constructed wetlands is gravel which did not show any negative effects on the performance of constructed wetlands. The gravel substrate was found efficient for sorption removal of the pharmaceuticals which are refractory to biodegradation but with relatively high hydrophobicity, for example, carbamazepine. However, at present, very few data are available on comprehensive understanding of the sorption performance of gravel and the associated mechanisms for the removal of pharmaceuticals from wastewater using constructed wetlands.

It has been reported in laboratory batch experiments that light expanded clay aggregate (LECA) is a good sorbent for the acidic pharmaceutical compounds such as clofibric acid and ibuprofen as well as the neutral pharmaceutical compounds such as carbamazepine (A.V. Dordio et al., 2009b). Dordio et al. (2007) compared three different substrate materials including LECA, expanded perlite, and sand for the removal of clofibric acid from water in laboratory. Their research results indicated that LECA exhibited a high sorption capacity for the removal of clofibric acid, while the expanded perlite only had a very limited sorption capacity and the sand did not show any sorption capacity at all. Considering the alkaline nature of LECA, electrostatic interactions in the case of the acidic pharmaceuticals have been hypothesized as being responsible for the affinities of these compounds towards LECA's surface. For the neutral compounds, van der Waals interactions may be more relevant. Dordio et al. (2009a) found that LECA also showed strong sorption for atenolol which was alkaline and positively charged. Electrostatic interactions cannot explain this phenomenon, but perhaps ion exchange may be responsible to some extent for the removal of atenolol by LECA.

Soil is another kind of popular substrate in constructed wetlands. Hussain et al. (2012) undertook some laboratory batch experiments to investigate the removal efficiencies of three antibiotics (monensin, salinomycin, and narasin) in two SF-CWs with different soil substrates. Higher removal efficiency was observed for the sandy soil when compared with the sandy loam soil. They explained that the higher relative hydraulic conductivity of the sandy soil could facilitate water movement within the soil profile resulting in a greater opportunity of soil-tocontaminants interactions. Whereas, the sandy clay loam soil which contained higher organic matter content showed a lower infiltration rate in the soil medium. However, their experimental results didn't indicate that the sorptive capability of the sandy soil was better than that of the sandy clay loam soil. Lertpaitoonpan et al. (2009) demonstrated that higher coefficient values $\left(K_{d}\right)$ could be found for the soils with higher organic carbon during the batch sorption process of sulfamethazine onto five soils (organic carbon content ranged from $0.1 \%$ to $3.8 \%$ ).

Recently, certain biosorbents such as rice husk, pine bark, and granulated cork have been considered as interesting alternatives to the common substrate materials in constructed wetlands due to their low cost, economical value of reuse, and easy disposal by incineration. A.V. Dordio et al., 2011b evaluated the sorption capacity of granulated cork to remove ibuprofen, carbamazepine and clofibric acid from water. Their laboratory experiments showed that the granulated cork presented good sorption qualities and the three pharmaceutical compounds were removed in the order of efficiency as: ibuprofen $>$ carbamazepine $>$ clofibric acid. In comparison with LECA, granulated cork had a much larger specific sorption capacity for all the target compounds.

Sorption kinetics and isotherms have been studied to describe the sorption process of pharmaceutical compounds to certain kinds of sorbent materials. Dordio et al. (2009a, 2010), A.V. Dordio et al., (2011b) observed that within the initial stage of $6 \mathrm{~h}$, more than half of the initial atenolol, carbamazepine, clofibric acid and ibuprofen could be removed via adsorption. Their laboratory experiment results indicated that the Freundlich equation modeled the experimental data better than the Langmuir equation. It reflected the significant role of the heterogeneity of the sorbent material as well as the possible lateral interactions between the sorbed molecules. Xu et al. (2009) also found that the Freundlich equation could well describe the adsorption process of their target pharmaceuticals in agricultural soils. The degradation of the target pharmaceuticals followed firstorder exponential decay kinetics with the adsorption affinity order as follows: triclosan $>$ clofibric acid $>$ naproxen $>$ diclofenac $>$ ibuprofen. Moreover, Yu et al. (2013) confirmed that the Freundlich equation and the first order decay kinetics fitted well the adsorption process of carbamazepine, gemfibrozil and triclosan onto three different sorbents such as Palmdale sand, Imperial Valley clay and Washington Palouse loam.

The $\mathrm{pH}$ of substrate materials could play an important role in the sorption capacity of substrate for pharmaceutical removal.Vasudevan et al. (2009) demonstrated that the overall sorption efficiency of ciprofloxacin could be influenced by a difference in soil pH. Hussain and Prasher (2011) observed an inverse relationship between the substrate $\mathrm{pH}$ and the sorption of three antibiotics (monensin, salinomycin, and narasin). Sassman and Lee (2007) also found an inverse correlation 
between the soil $\mathrm{pH}$ and the sorption coefficient of monensin and lasalocid. Lertpaitoonpan et al. (2009) explained that the $\mathrm{pH}$ of soil inversely influenced the sorption process of sulfamethazine due to the different ionization states of sulfamethazine under different $\mathrm{pH}$ conditions. At $\mathrm{pH}$ less than 7.4, hydrophobic partitioning was possibly responsible for the removal of the non-ionized form of sulfamethazine. At $\mathrm{pH}$ above 7.4 , the decrease in sorption of sulfamethazine was probably due to the predominance of the anionic form of sulfamethazine. Similar trend was also found for sulfonamides that the conversion of the neutral/cationic form of sulfonamides to the anionic form of sulfonamides above pH 7.5 led to a lower sorption to soils (Kurwadkar et al., 2007).

Competitive sorption phenomenon might take place if multiple pharmaceutical compounds and other wastewater pollutants are present in the water at the same time (Conkle et al., 2010; A.V. Dordio et al., 2009b). The sorption capacity of one pharmaceutical compound may be decreased by another pharmaceutical compound or/and other contaminants due to competition for preferred binding sites on the solid matrices in constructed wetlands. Therefore, this competition effect may be of concern in constructed wetland systems which are loaded with a wide range of pharmaceutical compounds.

\subsection{Plants}

Plants in constructed wetlands play a significant role in direct uptake of many organic pollutants in wastewater. In the cell membranes of plant roots, there are no specific transporters for the xenobiotic organic compounds like pharmaceuticals to move into the plants tissues, but the uptake and translocation of these compounds within plants can be simply driven by diffusion (Dietz and Schnoor, 2001; Dordio et al., 2011a; Dordio and Carvalho, 2013).

The diffusion process of pharmaceuticals into plant tissues is dependent on the physico-chemical characteristics of the compounds including the hydrophobicity (as expressed by logarithm octanol/ water partition coefficient $\log \mathrm{K}_{\mathrm{ow}}$ ), water solubility and concentration (Dordio and Carvalho, 2013; Stottmeister et al., 2003). In general, the pharmaceutical compounds characterized by a Log $\mathrm{K}_{\mathrm{ow}}$ in a moderate range of 0.5 to 3.5 are lipophilic enough to move through the lipid bilayer of plant cell membranes and also water soluble enough to travel into the cell fluids of plants (Dietz and Schnoor, 2001; Dordio and Carvalho, 2013; Schröder and Collins, 2002; Stottmeister et al., 2003). For instance, carbamazepine with a Log $\mathrm{K}_{\mathrm{ow}}$ of 2.45 has been reported to be readily taken up by the roots of Typha spp. and then transported from roots to stems and leaves, ultimately showing the largest extent of accumulation in leaves (A.V. Dordio et al., 2011a). In contrast to carbamazepine, for diclofenac whose Log $\mathrm{K}_{\mathrm{ow}}$ is 4.51 higher than 3.5, Zhang et al. (2012c) demonstrated that the compound had limited ability to be taken up and translocated within the roots and shoots of scirpus validus.

For the nonionizable, polar, highly water soluble organic compounds, Dettenmaier et al. (2009) found that caffeine could be taken up by plant roots and translocated to shoots, which is in agreement with the findings of Matamoros et al. (2012b) and D.Q. Zhang et al., 2013. Liu et al. (2013b) also observed a similar phenomenon for three antibiotics (ciprofloxacin $\mathrm{HCl}$, oxytetracycline $\mathrm{HCl}$ and sulfamethazine) which have the $\log \mathrm{K}_{\mathrm{ow}}$ values below 0.5 . The authors suggested that such highly water soluble organic compounds were most likely to be driven by the transpiration water stream in the plant uptake and the translocation within plant tissues. Further, they also found the positive correlation between the antibiotics concentrations and the accumulation levels of antibiotics inside the plants.

After being taken up into plant tissues, the internal pharmaceutical compounds might be degraded via the metabolism processes (phytodegradation). The metabolism processes might include a series of biochemical reactions such as transformation of parent organic pollutants, conjugation of metabolites with macromolecules, and incorporation of conjugated products into plant cell walls and vacuoles (Dordio and Carvalho, 2013; Reinhold et al., 2010). A.V. Dordio et al., 2011a detected a metabolite (10, 11-dihydro-10, 11-epoxycarbamazepine) of carbamazepine in the leaf tissues of Typha spp. indicating the occurrence of carbamazepine metabolism inside the plant tissues. Liu et al. (2013b) found the conversion of ciprofloxacin $\mathrm{HCl}$ and oxytetracycline $\mathrm{HCl}$ to their epimers such as tetracycline, chlortetracycline, enrofloxacin and ofloxacin in plants.

Plants in constructed wetlands play another important role in stimulating the development and activities of microbial populations which are supported by the rhizodeposition products (exudates, mucigels, dead cell material, etc.), causing various biological processes to occur in the rhizosphere (Calheiros et al., 2009; Cronk, 1996; Stottmeister et al., 2003). Matamoros et al. (2012b) found that the predominant removal process of ibuprofen was microbial degradation which was very possibly associated with the biofilms on the surface of plant roots. In addition to the biological processes, certain plant exudates may also function as catalytic agents for the degradation of organic compounds (Dordio and Carvalho, 2013). Most plant species in constructed wetlands are able to release oxygen around their root tips and on young laterals (Stottmeister et al., 2003). The oxygen released in rhizosphere can also promote the oxidative chemical processes of contaminants in wastewater (Sundaravadivel and Vigneswaran, 2001) and favor the development of aerobic microorganisms in the rhizosphere inducing more efficient biodegradation processes (Cronk, 1996; Dordio and Carvalho, 2013). The continuous release of oxygen from the root zones of plants might counterbalance the chemical and biological oxygen consumption in the rhizosphere.

It has been reported that the presence of plants in constructed wetlands played a positive role in the removal of some certain pharmaceuticals such as diclofenac, ibuprofen, ketoprofen, naproxen, salicylic acid, amoxicillin, ampicillin, erythromycin, sulfadiazine, sulfamethazine, sulfamethoxazole, atenolol, clofibric acid, carbamazepine and caffeine (Dordio et al., 2010, 2009a; Hijosa-Valsero et al., 2011a, 2011b, 2010b, 2011c; Xian et al., 2010; Matamoros et al., 2012a, 2012; Zhang et al., 2012a; Zhang et al., 2012b, 2011). Several plant species have been reported for use in pharmaceutical wastewater treatment wetlands (Table 5) among which the most popular plants are Phragmites australis, Typha spp. Typha angustifolia and Typha latifolia. The experiment results of Hijosa-Valsero et al. (2010b, 2011c) indicated that P. australis had a better performance than T. angustifolia. However, Dordio et al. (2009a) observed the superiority of Typha spp. compared to the P. australis due to the higher transpiration rate of Typha spp. Zarate et al. (2012) investigated the accumulation of triclosan inside the plant tissues (roots and shoots) and their results showed the species-specific differences among different vegetations (Pontederia cordata and Sagittaria graminea), indicating the important influences of plant species on the pharmaceutical removal.

Toxicity to plants caused by pharmaceuticals is an important issue when considering the functions of plants for pharmaceutical removal. The toxicity can be evaluated through analyzing the root activity, the relative growth rate (RGR) of plant and the concentrations of photosynthetic pigments such as carotenoid and chlorophyll in plants tissues (Dordio et al., 2011a, A.V. Dordio et al., 2011a, 2009a; Liu et al., 2013b). In addition, the alteration of antioxidant enzyme activities in plant tissues can also serve as an indication of the phytotoxicity caused by pharmaceutical compounds. The antioxidant enzymes like catalase (CAT), superoxide dismutase (SOD), guaiacol peroxidase (GPX) and ascorbate peroxidase (APX) have been reported for the plants which are subjected to pharmaceutical stress (Dordio et al., 2011a, A.V. Dordio et al., 2011a, 2009a; Liu et al., 2013b).

\subsection{Microbes}

Microbes in constructed wetlands usually play the main role in the processes of transformation and mineralization of nutrients and organic pollutants (Kadlec and Wallace, 2009; Sundaravadivel and Vigneswaran, 2001; Truu et al., 2009; Zhu et al., 2010, 2011, 2013). Biodegradation of organic compounds by microbes in constructed 


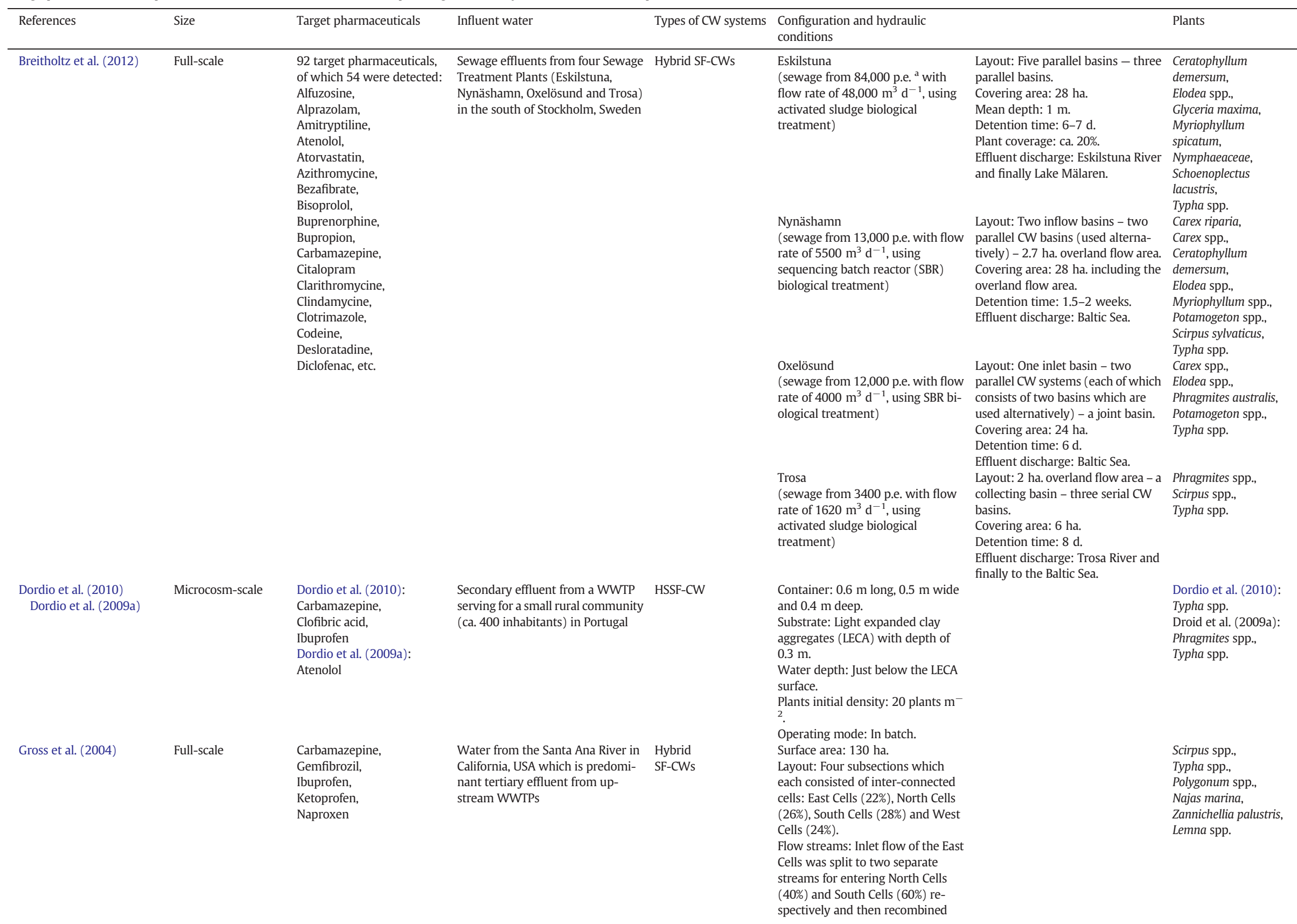




\begin{tabular}{|c|c|c|c|c|c|c|}
\hline $\begin{array}{l}\text { Lee et al. (2011) } \\
\text { Park et al. (2009) }\end{array}$ & Full-scale & $\begin{array}{l}\text { Lee et al. (2011): } \\
\text { Atenolol, } \\
\text { Caffeine, } \\
\text { Carbamazepine, } \\
\text { Diclofenac, } \\
\text { Glimepiride, } \\
\text { lbuprofen, } \\
\text { Naproxen, } \\
\text { Sulfamethoxazole } \\
\text { Park et al. (2009): } \\
\text { Atenolol, } \\
\text { Carbamazepine, } \\
\text { Diazepam, } \\
\text { Diclofenac, } \\
\text { Dilantine, } \\
\text { Sulfamethoxazole, } \\
\text { Triclosan, } \\
\text { Naproxen }\end{array}$ & $\begin{array}{l}\text { Wastewater effluent from the } \\
\text { Damyang wastewater treatment } \\
\text { plant in Korea }\left(1800 \mathrm{~m}^{3} \mathrm{~d}^{-1}\right)\end{array}$ & Hybrid SF-CWs & $\begin{array}{l}\text { Layout: Two basins in series } \\
\text { (Acorus wetland and Typha } \\
\text { wetland); } 30 \mathrm{~m} \text { wide, } 120 \mathrm{~m} \text { long } \\
\text { and } 0.13 \mathrm{~m} \text { deep. } \\
\text { HRT: } 6 \mathrm{~h} \text {. }\end{array}$ & $\begin{array}{l}\text { Acorus spp., } \\
\text { Typha spp. }\end{array}$ \\
\hline $\begin{array}{l}\text { Llorens et al. (2009) } \\
\text { Matamoros et al. (2008b) }\end{array}$ & Full-scale & $\begin{array}{l}\text { Llorens et al. (2009): } \\
\text { Carbamazepine, } \\
\text { Clofibric acid, } \\
\text { Diclofenac, } \\
\text { Ibuprofen, } \\
\text { Ketoprofen, } \\
\text { Naproxen } \\
\text { Matamoros et al. (2008b): } \\
\text { Carbamazepine, } \\
\text { Clofibric acid, } \\
\text { Diclofenac, } \\
\text { Flunixin } \\
\text { Ibuprofen, } \\
\text { Ketoprofen, } \\
\text { Naproxen }\end{array}$ & $\begin{array}{l}\text { Part of secondary effluent from the } \\
\text { Granollers urban WWTP in Spain } \\
\left(100 \mathrm{~m}^{3} \mathrm{~d}^{-1}\right)\end{array}$ & SF-CW & $\begin{array}{l}\text { Surface area: } 1 \text { ha. (maximum } \\
\text { length and width of around } 189 \mathrm{~m} \\
\text { and } 53 \mathrm{~m} \text { respectively). } \\
\text { Water depth: } 0.3-0.4 \mathrm{~m} \text { for planted } \\
\text { shallow zones; } 1.5 \mathrm{~m} \text { for unplanted } \\
\text { deep zones. } \\
\text { HRT: } 1 \text { month. } \\
\text { Hydraulic loading rate (HLR): } \\
1 \mathrm{~cm} \mathrm{~d}^{-1} \text {. } \\
\text { Effluent discharge: Congost River. }\end{array}$ & $\begin{array}{l}\text { Phragmites australis, } \\
\text { Typha latifolia }\end{array}$ \\
\hline $\begin{array}{l}\text { Matamoros and Salvadó } \\
\text { (2012) }\end{array}$ & Full-scale & $\begin{array}{l}\text { Caffeine, } \\
\text { Carbamazepine, } \\
\text { Diclofenac, } \\
\text { Ibuprofen, } \\
\text { Ketoprofen, } \\
\text { Naproxen, } \\
\text { Triclosan }\end{array}$ & $\begin{array}{l}\text { Secondary effluent from the } \\
\text { Empuriabrava WWTP in Spain } \\
\left(3700 \mathrm{~m}^{3} \mathrm{~d}^{-1} \text {, seasonally ranging }\right. \\
\left.\text { from } 2300 \mathrm{~m}^{3} \mathrm{~d}^{-1} \text { to } 6400 \mathrm{~m}^{3} \mathrm{~d}^{-1}\right)\end{array}$ & $\begin{array}{l}\text { Hybrid pond and SF- } \\
\text { CW in series }\end{array}$ & $\begin{array}{l}\text { Ponds (two parallel cells): } 2 \text { ha. } \\
\text { each; } 1 \mathrm{~m} \text { deep; HRT was } 4 \mathrm{~d} \text {. } \\
\text { SF-CWs (three parallel deep cells } \\
\text { and a large shallow cell in series): } \\
\text { The deep cells were } 0.8 \text { ha. and } \\
0.5 \mathrm{~m} \text { deep each; the shallow cell } \\
\text { was } 4.5 \text { ha. and } 0.2 \text { m deep; HRT } \\
\text { was } 8.5 \mathrm{~d} \text {. } \\
\text { Effluent discharge: Pumped into an } \\
18 \text { ha. artificial pond to maintain } \\
\text { the flooding of the marshlands. }\end{array}$ & $\begin{array}{l}\text { Phragmites australis, } \\
\text { Typha latifolia }\end{array}$ \\
\hline Matamoros et al. (2012a) & Full-scale & $\begin{array}{l}\text { Caffeine, } \\
\text { Carbamazepine, } \\
\text { Diclofenac, } \\
\text { Furosemide, } \\
\text { Ibuprofen, } \\
\text { Ketoprofen, } \\
\text { Naproxen, } \\
\text { Triclosan }\end{array}$ & $\begin{array}{l}\text { Water from two feeding rivers } \\
\text { in Denmark: Aarhus River } \\
\left(0.96 \mathrm{~m}^{3} \mathrm{~s}^{-1}\right) \text { and Lyngbygaard } \\
\text { River }\left(1.03 \mathrm{~m}^{3} \mathrm{~s}^{-1}\right) \text { which were } \\
\text { subjected to the discharges of the } \\
\text { WWTPs located upstream }\end{array}$ & SF-CW & $\begin{array}{l}\text { Surface area: } 100 \text { ha. } \\
\text { Water depth: } 0.05 \mathrm{~m} \text { to } 2 \mathrm{~m} \text {. } \\
\text { HRT: } 3 \text { to } 20 \mathrm{~d} \text { (average } 7 \mathrm{~d} \text { ). } \\
\text { Effluent discharge: Lake Brabrand. }\end{array}$ & $\begin{array}{l}\text { Nymphaea alba, } \\
\text { Schoenoplectus } \\
\text { lacustris }\end{array}$ \\
\hline Reif et al. (2011) & Pilot-scale & $\begin{array}{l}\text { Carbamazepine, } \\
\text { Diclofenac, } \\
\text { Ibuprofen, } \\
\text { Naproxen }\end{array}$ & $\begin{array}{l}\text { Sewage effluents from a membrane } \\
\text { reactor (MBR) and an activated } \\
\text { sludge (AS) system in UK }\end{array}$ & VSSF-CW & $\begin{array}{l}\text { Feeding mode: } \mathrm{CW} \text { beds were fed } 8 \\
\text { times per day for } 10 \mathrm{~min} \text { at } \\
125 \mathrm{~mL} \text { min }^{-1} \text {. } \\
\text { Depth: } 1 \mathrm{~m} \text {; }\end{array}$ & Phragmites spp. \\
\hline
\end{tabular}


Table 3 (continued)

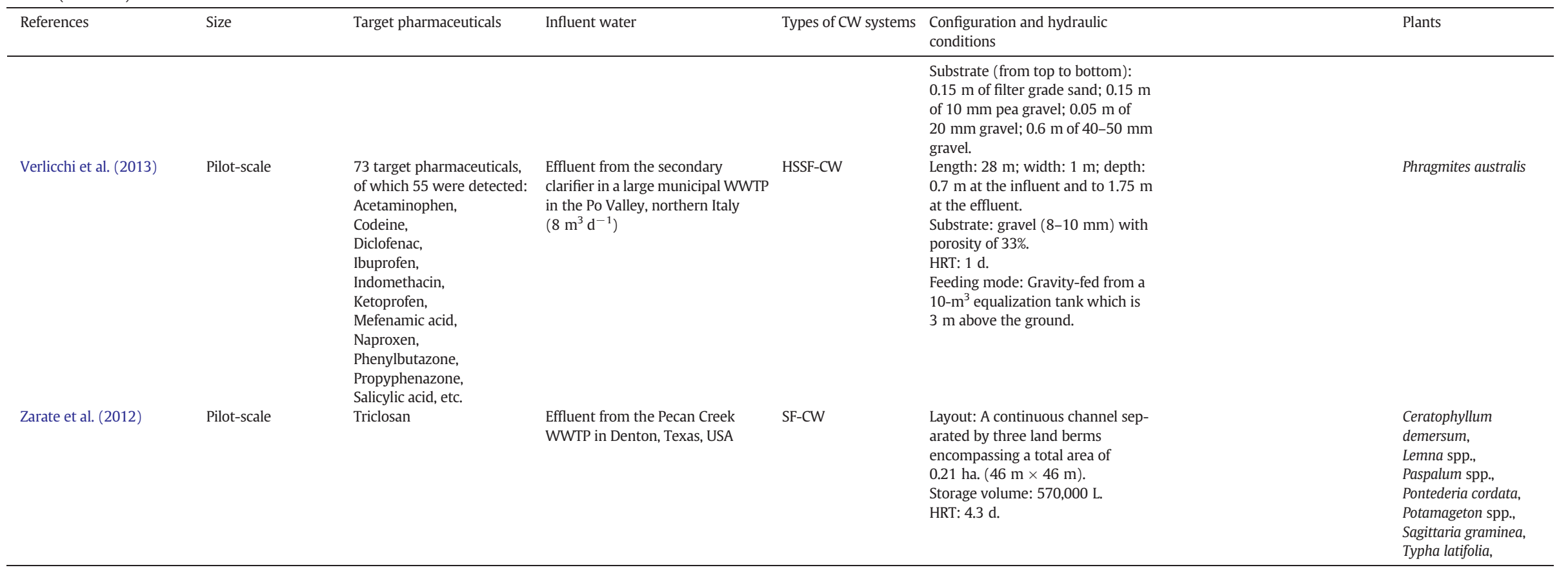

Notes: ${ }^{a}$ p.e.: people equivalent. 
Table 4

Removal efficiencies of pharmaceuticals in constructed wetlands applied as a wastewater polishing treatment system.

\begin{tabular}{|c|c|c|c|c|}
\hline Pharmaceutical compounds & SF-CW & HSSF-CW & VSSF-CW & Hybrid CW system \\
\hline \multicolumn{5}{|l|}{ Analgesic/anti-inflammatory } \\
\hline Acetaminophen & & $45(12)$ & & \\
\hline Buprenorphine & & & & -2956 to $58(7)$ \\
\hline Codeine & & $57(12)$ & & $29-75(7)$ \\
\hline Diclofenac & $\begin{array}{l}85 \text { (1); } 3 \text { (2); } 96 \text { (summer, 3); } \\
73 \text { (winter, 3) }\end{array}$ & $5(12)$ & $78-79(6)$ & $\begin{array}{l}24-36(7) ; 86-98(8) \\
38-85(9) ; 62(10)\end{array}$ \\
\hline Ibuprofen & $\begin{array}{l}96 \text { (1); } 19 \text { (2); } 96 \text { (summer, 3); } \\
95 \text { (winter, 3) }\end{array}$ & $\begin{array}{l}\text { 82-96 (winter-summer, planted, 4); } \\
\text { 74-91 (winter-summer, unplanted, 4); } \\
28 \text { (12) }\end{array}$ & $66-72(6)$ & $\begin{array}{l}5-88(7) ; 79-97(8) ; \\
55(10) ; 59(11)\end{array}$ \\
\hline Indomethacin & & $45(12)$ & & \\
\hline Ketoprofen & $\begin{array}{l}98 \text { (1); } 99 \text { (summer, } 3 \text { ); } \\
97 \text { (winter, 3) }\end{array}$ & $18(12)$ & & 3-56 (16); 97-99 (8) \\
\hline Mefenamic acid & & $20(12)$ & & \\
\hline Naproxen & $\begin{array}{l}72 \text { (1); } 4 \text { (2); } 92 \text { (summer, 3); } \\
52 \text { (winter, 3) }\end{array}$ & $36(12)$ & $39-45(6)$ & $\begin{array}{l}34-75(7) ; 72-96(8) \\
71-82(9) ; 67(10)\end{array}$ \\
\hline Phenylbutazone & & $55(12)$ & & \\
\hline Propyphenazone & & $-19(12)$ & & \\
\hline Orphenadrine & & & & -19 to $54(7)$ \\
\hline Salicylic acid & & $7(12)$ & & \\
\hline Tramadol & & & & -12 to $26(7)$ \\
\hline \multicolumn{5}{|l|}{ Antiallergic drugs } \\
\hline Desloratidin & & & & $47-72(7)$ \\
\hline Diphenhydramine & & & & $46-50(7)$ \\
\hline Fexofenadine & & & & -26 to $28(7)$ \\
\hline \multicolumn{5}{|l|}{ Antibiotics } \\
\hline Azithromycine & & $86(12)$ & & -350 to $2(7)$ \\
\hline Ciprofloxacin & & $67(12)$ & & \\
\hline Clarithromycine & & $7(12)$ & & -58 to $58(7)$ \\
\hline Clindamycine & & & & -103 to $13(7)$ \\
\hline Enoxacin & & $38(12)$ & & \\
\hline Erythromycin & & $-78(12)$ & & \\
\hline Metronidazole & & $100(12)$ & & \\
\hline Nifuroxazide & & $100(12)$ & & \\
\hline Norfloxacin & & $52(12)$ & & \\
\hline Ofloxacin & & $84(12)$ & & \\
\hline Roxithromycin & & $-43(12)$ & & \\
\hline Spiramycin & & $58(12)$ & & \\
\hline Sulfadiazine & & $-17(12)$ & & \\
\hline Sulfamethazine & & $100(12)$ & & \\
\hline Sulfamethoxazole & & $16(12)$ & & -104 to $12(7) ; 28-53(9) ; 23(10)$ \\
\hline Tilmicosin & & $51(12)$ & & \\
\hline Triclosan & $50(2)$ & & & 74-93 (8); 67-99 (9) \\
\hline Trimethoprim & & $38(12)$ & & $25-86(7)$ \\
\hline \multicolumn{5}{|l|}{ Antidiabetics } \\
\hline Glibenclamide & & $23(12)$ & & \\
\hline Glimepiride & & & & $20(10)$ \\
\hline \multicolumn{5}{|l|}{ Antidysenterics } \\
\hline Loperamide & & & & $38(7)$ \\
\hline \multicolumn{5}{|l|}{ Antifungals } \\
\hline Clotrimazol & & & & $-18(7)$ \\
\hline Fluconazole & & & & -40 to $43(7)$ \\
\hline \multicolumn{5}{|l|}{ Antihypertensives } \\
\hline Diltiazem & & & & $30-88(7)$ \\
\hline Dipyridamol & & & & $80-100(7)$ \\
\hline Eprosartan & & & & $24-75(7)$ \\
\hline Hydrochlorothiazide & & $63(12)$ & & \\
\hline Ibersartan & & & & $3-58(7)$ \\
\hline Telmisartan & & & & $4-87(7)$ \\
\hline Verapamil & & & & $69-84(7)$ \\
\hline \multicolumn{5}{|l|}{ Anti-senile dementia drugs } \\
\hline Memantin & & & & $5-32(7)$ \\
\hline \multicolumn{5}{|l|}{ Barbiturates } \\
\hline Butalbital & & $42(12)$ & & \\
\hline Pentobarbital & & $32(12)$ & & \\
\hline Phenobarbital & & $17(12)$ & & \\
\hline \multicolumn{5}{|l|}{ Beta-agonists } \\
\hline Clenbuterol & & $11(12)$ & & \\
\hline Salbutamol & & $100(12)$ & & \\
\hline Terbutaline & & & & -11 to $83(7)$ \\
\hline
\end{tabular}


Table 4 (continued)

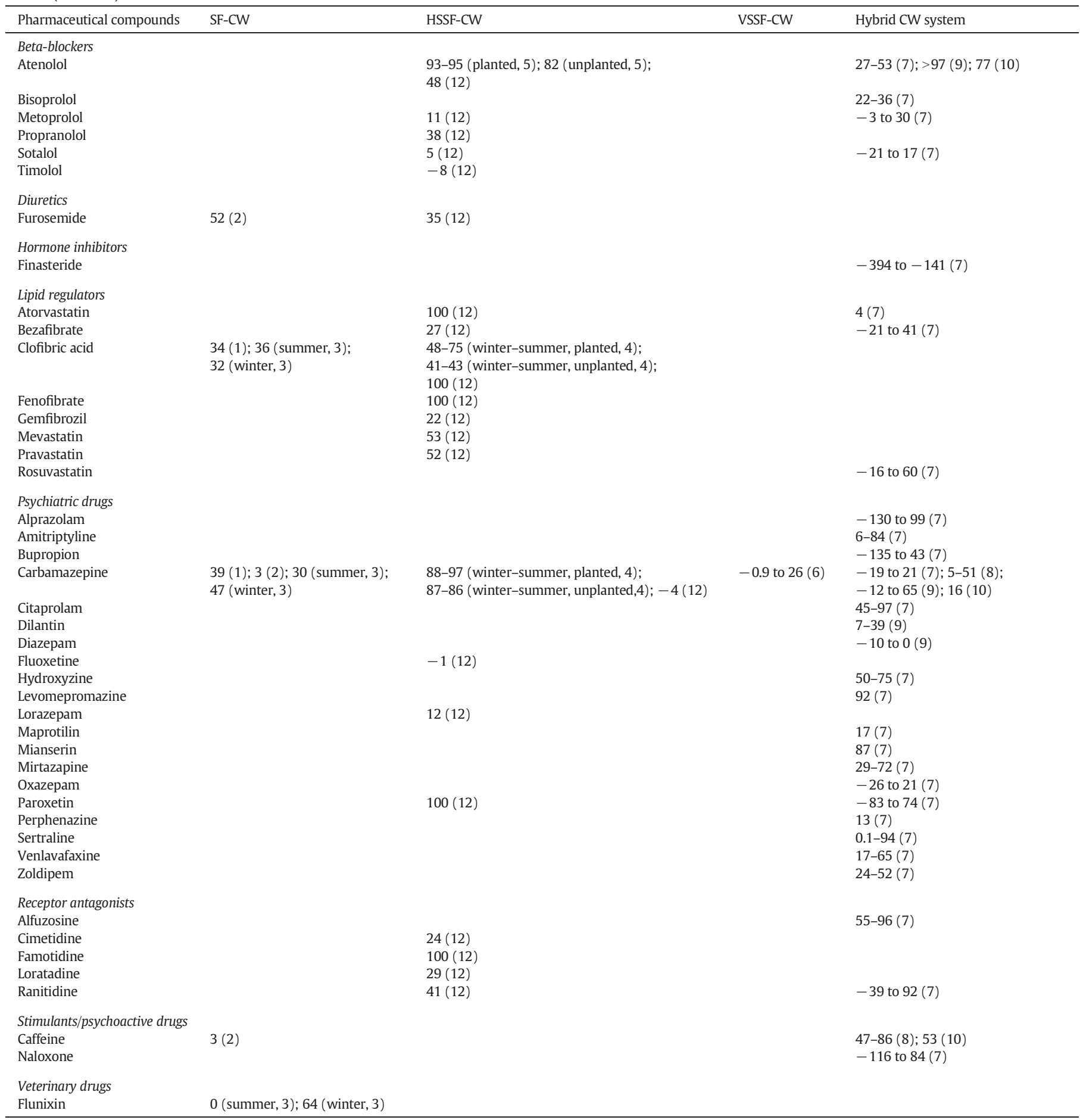

Notes: 1: Llorens et al. (2009); 2: Matamoros et al. (2012a); 3: Matamoros et al. (2008b); 4: Dordio et al. (2010); 5: Dordio et al. (2009a); 6: Reif et al. (2011); 7: Breitholtz et al. (2012); 8 Matamoros and Salvadó (2012); 9: Park et al. (2009); 10: Lee et al. (2011); 11: Gross et al. (2004); 12: Verlicchi et al. (2013).

wetlands can occur under both the aerobic and the anaerobic conditions involving the activities of various microorganisms such as heterotrophic bacteria, autotrophic bacteria, fungi (basidiomycetes and yeasts), and specific protozoa (Kadlec and Wallace, 2009).

An important factor strongly influencing the microbial degradation process is the chemical structures of the organic compounds (Dua et al., 2002; Reddy and DeLaune, 2008). For those organic compounds with simple structures possessing high water solubility and low adsorptivity, they could be readily degraded by microorganisms.
It is attributable to the usual similarity of these organic compounds to the naturally occurring compounds which are commonly used as energy sources by microorganisms. In contrast, for those xenobiotic organic compounds (including pharmaceuticals) that have the very different structures from the naturally occurring compounds, they may be slowly degraded by microorganisms possibly due to the lack of suitable degrading genes in microorganisms. However, it does not mean that the non-specific enzymes are not able to help degrade the xenobiotic organic compounds. The degradation of such organic 
Table 5

Reported plant species in constructed wetlands for the removal of pharmaceuticals.

\begin{tabular}{|c|c|c|}
\hline Common name & Latin name & Sources \\
\hline \multicolumn{3}{|l|}{ Shrubs } \\
\hline Grey willow & Salix atrocinerea & Hijosa-Valsero et al. (2011c, 2010a) \\
\hline \multicolumn{3}{|l|}{ Emergent plants } \\
\hline Arrowhead & Sagittaria graminea, & Zarate et al. (2012) \\
\hline \multirow[t]{2}{*}{ Bulrush } & Scirpus spp. & Breitholtz et al. (2012); Gross et al. (2004) \\
\hline & Schoenoplectus lacustris & Breitholtz et al. (2012); Matamoros et al. (2012a) \\
\hline \multirow[t]{2}{*}{ Common reed } & Phragmites spp. & Breitholtz et al. (2012); Dordio et al. (2009a); Reif et al. (2011) \\
\hline & Phragmites australis & $\begin{array}{l}\text { Ávila et al. (2010, 2013); Breitholtz et al. (2012); Carvalho et al. (2013); Conkle et al. (2008); } \\
\text { Hijosa-Valsero et al. (2011a, 2011b, 2011c, 2010b); Llorens et al. (2009); Matamoros and } \\
\text { Salvadó, (2012); Matamoros et al. (2008a, 2008b, 2007); Matamoros and Bayona (2006); } \\
\text { Matamoros et al. (2005); Ranieri et al. (2011); Reyes-Contreras et al. (2012); Verlicchi et al. } \\
\text { (2013) }\end{array}$ \\
\hline Calamus & Acorus spp. & Lee et al. (2011); Park et al. (2009) \\
\hline Cattail & Typha spp. & $\begin{array}{l}\text { Anderson et al. (2013); Breitholtz et al. (2012); Dordio et al. (2010, 2009a); Gross et al. } \\
\text { (2004); Lee et al., 2011; Park et al. (2009) }\end{array}$ \\
\hline Narrowleaf cattail/lesser bulrush & Typha angustifolia & $\begin{array}{l}\text { Hijosa-Valsero et al. (2011a, 2011c, 2010b); Reyes-Contreras et al. (2012); Zhang et al. } \\
\text { (2012a, 2012b, 2011) }\end{array}$ \\
\hline Broadleaf cattail/common bulrush & Typha latifolia & $\begin{array}{l}\text { Hussain et al. (2012, 2011); Hijosa-Valsero et al. (2011c, 2010a); Llorens et al. (2009); } \\
\text { Matamoros and Salvadó (2012); Matamoros et al. (2008b); Ranieri et al. (2011); Zarate et al. } \\
\text { (2012) }\end{array}$ \\
\hline Dallis grass & Paspalum spp., & Zarate et al. (2012) \\
\hline Fountain grass & Pennisetum & Liu et al. (2013a) \\
\hline Reed canarygrass & Phalaris arundinacea & Hussain et al. $(2012,2011)$ \\
\hline Reed mannagrass & Glyceria maxima & Breitholtz et al. (2012) \\
\hline Ryegrass & Lolium & Xian et al. (2010) \\
\hline Sedge & Carex spp. & Breitholtz et al. (2012) \\
\hline Variegated greater pond sedge & Carex riparia & Breitholtz et al. (2012) \\
\hline Soft rush & Juncus effusus & Reyes-Contreras et al. (2011) \\
\hline Wood clubrush & Scirpus sylvaticus & Breitholtz et al. (2012) \\
\hline Pickerelweed & Pontederia cordata & Zarate et al. (2012) \\
\hline \multicolumn{3}{|l|}{ Submergent plants } \\
\hline Coontail/Hornwort & Ceratophyllum demersum & Breitholtz et al. (2012); Zarate et al. (2012) \\
\hline Eurasian watermilfoil & Myriophyllum spicatum & Breitholtz et al. (2012) \\
\hline Horned pondweed & Zannichellia palustris & Gross et al. (2004) \\
\hline Pondweed & Potamogeton spp. & Breitholtz et al. (2012); Zarate et al. (2012) \\
\hline Spiny naiad & Najas marina & Gross et al. (2004) \\
\hline Water milfoil & Myriophyllum spp. & Breitholtz et al. (2012) \\
\hline Water weed & Elodea spp. & Breitholtz et al. (2012) \\
\hline \multicolumn{3}{|l|}{ Floating plants } \\
\hline Duckweed & Lemna spp. & Gross et al. (2004); Zarate et al. (2012) \\
\hline Common duckweed/lesser duckweed & Lemna minor & Hijosa-Valsero et al. (2011c, 2010a) \\
\hline Pennywort & Hydrocottle spp. & Conkle et al. (2008) \\
\hline Smartweed & Polygonum spp. & Gross et al. (2004) \\
\hline \multicolumn{3}{|l|}{ Rooted floating leaves } \\
\hline Water lily & Nymphaeaceae & Breitholtz et al. (2012) \\
\hline White lotus & Nymphaea alba & Matamoros et al. (2012a) \\
\hline
\end{tabular}

compounds by non-specific enzymes may still take place at a slower rate via co-metabolism reactions which do not support the microbial growth (Seffernick and Wackett, 2001). In addition, the degradation of xenobiotic organic compounds by microorganisms in constructed wetlands may also be influenced by the substrate, vegetation, oxygen and redox potential, temperature, $\mathrm{pH}$, nutrient available, and presence of toxic substances (Calheiros et al., 2009; Li et al., 2010; Reddy and DeLaune, 2008; Salomo et al., 2009; Truu et al., 2009).

In the reviewed research work, aerobic biodegradation was thought to be the main contribution to the microbial degradation process of ibuprofen (Ávila et al., 2010, 2013; Hijosa-Valsero et al., 2010a, 2010b, 2011c; Matamoros et al., 2008a, 2005; Reyes-Contreras et al., 2012), salicylic acid (Hijosa-Valsero et al., 2010b, 2011c; Reyes-Contreras et al., 2012) and sulfamethoxazole (Hijosa-Valsero et al., 2011a), whereas anaerobic biodegradation was thought to be responsible for naproxen (Ávila et al., 2010) and caffeine (Hijosa-Valsero et al., 2010b; HijosaValsero et al., 2011c; Reyes-Contreras et al., 2012). However, regarding the biodegradation of diclofenac, there exist two different points of views and they are: 1 ) high redox potential could promote the removal of diclofenac (Ávila et al., 2013; Hijosa-Valsero et al., 2010b, 2011c); 2) the removal efficiency of diclofenac could be enhanced under anaerobic conditions (Ávila et al., 2010). All the removal mechanisms stated above were inferred from the reported experiment results and do not include explicit demonstrations. Thus, it is still difficult to describe the actual removal mechanisms of pharmaceuticals to date.

Certain pharmaceutical compounds (for example, ibuprofen) which contain an asymmetric carbon atom (also known as a chiral carbon atom) usually have two enantiomeric forms $\left(S_{\text {form }}\right.$ and $\left.R_{\text {form }}\right)$. The enantiomeric fraction ( $E F=S_{\text {form }} /\left(S_{\text {form }}+R_{\text {form }}\right)$ ) (Hijosa-Valsero et al., 2010a; Matamoros et al., 2009b) which is compound-dependent can be used for the evaluation on the occurrence of enantioselective degradation in wastewater treatment system through investigating the change in the trend of EF. Enantioselective degradation of ibuprofen was reported by Matamoros et al. (2009b) that the $S_{\text {form }}$ ibuprofen was degraded faster than the $\mathrm{R}_{\text {form }}$ ibuprofen under aerobic conditions (in VSSF-CW) inducing EF to decrease. This phenomenon was in agreement with the findings of Hijosa-Valsero et al. (2010a). However, EF didn't change during wastewater treatment under anaerobic conditions (in HSSF-CW) due to the similar degradation rates of $\mathrm{S}_{\text {form }}$ ibuprofen and the $\mathrm{R}_{\text {form }}$ ibuprofen. Owing to the different ibuprofen EF trends under aerobic and anaerobic conditions, it can be deduced which kind of metabolic pathway (aerobic biodegradation or 
anaerobic degradation) plays a predominant role in the removal of ibuprofen in constructed wetlands.

Evaluation of the biodegradation intermediates of pharmaceuticals in constructed wetlands is also important for elucidating the microbial degradation mechanisms, but available data are very few at present. Matamoros et al. (2008a) studied the behavior of two biodegradation intermediates of ibuprofen including carboxy-ibuprofen (CA-IBP) and hydroxy-ibuprofen (OH-IBP) in a HSSF-CW system to assess the relative contribution of aerobic and anaerobic pathways to the ibuprofen biodegradation. Their findings indicated that the CA-IBP and OH-IBP only contributed to $5 \%$ of the degraded IBP and there was negligible accumulation of these two kinds of intermediates in the microcosms. The observation was possibly due to the similar kinetics for their consumption and formation. However, both CA-IBP and OH-IBP can be produced under aerobic conditions and only CA-IBP can be detected under anaerobic conditions (Zwiener et al., 2002). Through the analysis on the ibuprofen removal percentage attributable to the aerobic and anaerobic pathways using Eqs. (1) and (2), the authors suggested that the aerobic metabolic pathways predominated for the removal of ibuprofen.

Aerobic-ratio $=\left(\mathrm{C}_{\mathrm{OH}-\mathrm{IBP}}+\mathrm{xC}_{\mathrm{CA}-\mathrm{IBP}}\right) /\left(\mathrm{C}_{\mathrm{OH}-\mathrm{IBP}}+\mathrm{C}_{\mathrm{CA}-\mathrm{IBP}}\right)$

Anaerobic-ratio $=\mathrm{yC}_{\mathrm{CA}-\mathrm{IBP}} /\left(\mathrm{C}_{\mathrm{OH}-\mathrm{IBP}}+\mathrm{C}_{\mathrm{CA}-\mathrm{IBP}}\right)$

where:

$\mathrm{C}_{\mathrm{CA}-\mathrm{IBP}}$ - Concentration of CA-IBP

$\mathrm{C}_{\mathrm{OH}}-\mathrm{IBP}-$ Concentration of $\mathrm{OH}-\mathrm{IBP}$

$\mathrm{x}$ - Percentage of CA-IBP obtained in the aerobic pathways, $0.4 \%$

$\mathrm{y}$ - Percentage of CA-IBP obtained in the anaerobic pathways, $1.8 \%$.

Feeding pharmaceutical contaminants to constructed wetland may affect the development and activities of microbes in constructed wetlands. Weber et al. (2011) assessed the influence of ciprofloxacin on the distribution of bacterial communities in some mesocosmscale constructed wetlands planted with $P$. australis. Their experimental results showed that the presence of ciprofloxacin had an initial adverse effect on the bacterial communities in the constructed wetlands, thereby reducing their ability to assimilate anthropogenic carbon-based compounds. However, after a 2-5 week acclimation period, the bacterial communities could return to their normal functionality. Helt et al. (2012) investigated the effect of ciprofloxacin on the antibiotic resistance of the interstitial bacterial community (total culturable heterotrophs) and the selected fecal indicators (Escherichia coli and Enterococcus spp.) in the same constructed wetlands as those used by Weber et al. (2011). Helt et al. (2012) found that the antibiotic resistance level of the interstitial bacteria community peaked at 7 days following the ciprofloxacin exposure and then decreased thereafter, and also observed that the antibiotic resistance level of the fecal indicators increased.

\section{Conclusions}

Constructed wetlands are now attracting increasing attention to their application for the removal of pharmaceutical contaminants from wastewater. Based on the findings of published work on the feasibility of constructed wetlands as a means to treat pharmaceutical contaminants in wastewater, one can deduce that constructed wetlands hold great potential of being used as an alternative secondary wastewater treatment system or as a wastewater polishing treatment system. Nevertheless, it remains true that scarce data at present cannot provide very persuasive demonstrations for the performance and effectiveness of constructed wetlands in these applications. There are knowledge gaps in several aspects including the removal efficiencies of pharmaceuticals in constructed wetlands, the removal mechanisms involved, the toxicity to constructed wetlands caused by pharmaceuticals, and the influences of certain important parameters (configuration design, hydraulic mode, temperature and seasonality, $\mathrm{pH}$, oxygen and redox potential, etc.). Moreover, most studies were undertaken at a microcosm-scale or mesocosmscale. Therefore, further research is required to provide more comprehensive and convincing evidences in larger laboratory-scale, pilot-scale or full-scale constructed wetlands. In addition, the current constructed wetlands applied for the treatment of pharmaceutical pollutants in wastewater are mainly the conventional constructed wetlands including SF-CWs, HSSF-CWs, VSSF-CWs, and hybrid CW system. Some novel constructed wetlands such as the tidal flow constructed wetlands, anti-sized constructed wetlands, and the dewatered alum sludge based constructed wetlands can also be investigated.

\section{Acknowledgments}

This work was financially supported by the Maritime Research Centre (MRC), the Advanced Environmental and Biotechnology Centre (AEBC), and the Nanyang Environment and Water Research Institute (NEWRI), Nanyang Technological University, Singapore. Guibing Zhu is funded by the National Natural Science Foundation of China (No. 41322012).

\section{References}

Anderson JC, Carlson JC, Low JE, Challis JK, Wong CS, Knapp CW, et al. Performance of a constructed wetland in Grand Marais, Manitoba, Canada: removal of nutrients, pharmaceuticals, and antibiotic resistance genes from municipal wastewater. Chem Cent J 2013;7.

Ávila C, Pedescoll A, Matamoros V, Bayona JM, García J. Capacity of a horizontal subsurface flow constructed wetland system for the removal of emerging pollutants: an injection experiment. Chemosphere 2010;81:1137-42.

Ávila C, Reyes C, Bayona JM, García J. Emerging organic contaminant removal depending on primary treatment and operational strategy in horizontal subsurface flow constructed wetlands: influence of redox. Water Res 2013;47:315-25.

Bendz D, Paxéus NA, Ginn TR, Loge FJ. Occurrence and fate of pharmaceutically active compounds in the environment, a case study: Höje River in Sweden. J Hazard Mater 2005; 122:195-204.

Breitholtz M, Näslund M, Stråe D, Borg H, Grabic R, Fick J. An evaluation of free water surface wetlands as tertiary sewage water treatment of micro-pollutants. Ecotoxicol Environ Saf 2012;78:63-71.

Calheiros CSC, Duque AF, Moura A, Henriques IS, Correia A, Rangel AOSS, et al. Substrate effect on bacterial communities from constructed wetlands planted with Typha latifolia treating industrial wastewater. Ecol Eng 2009;35:744-53.

Camacho-Muñoz D, Martín J, Santos JL, Aparicio I, Alonso E. Effectiveness of conventional and low-cost wastewater treatments in the removal of pharmaceutically active compounds. Water Air Soil Pollut 2012;223:2611-21.

Carballa M, Omil F, Lema JM, Llompart M, García-Jares C, Rodríguez I, et al. Behavior of pharmaceuticals, cosmetics and hormones in a sewage treatment plant. Water Res 2004;38:2918-26.

Carlsson C, Johansson A-K, Alvan G, Bergman K, Kühler T. Are pharmaceuticals potent environmental pollutants?: part I: environmental risk assessments of selected active pharmaceutical ingredients. Sci Total Environ 2006;364:67-87.

Carvalho PN, Araújo JL, Mucha AP, Basto MCP, Almeida CMR. Potential of constructed wetlands microcosms for the removal of veterinary pharmaceuticals from livestock wastewater. Bioresour Technol 2013;134:412-6.

Choudhary AK, Kumar S, Sharma C. Constructed wetlands: an option for pulp and paper mill wastewater treatment. Electron J Environ Agric Food Chem 2011;10:3023-37.

Clara M, Strenn B, Gans O, Martinez E, Kreuzinger N, Kroiss H. Removal of selected pharmaceuticals, fragrances and endocrine disrupting compounds in a membrane bioreactor and conventional wastewater treatment plants. Water Res 2005;39:4797-807.

Conkle JL, White JR, Metcalfe CD. Reduction of pharmaceutically active compounds by a lagoon wetland wastewater treatment system in Southeast Louisiana. Chemosphere 2008;73:1741-8.

Conkle JL, Lattao C, White JR, Cook RL. Competitive sorption and desorption behavior for three fluoroquinolone antibiotics in a wastewater treatment wetland soil. Chemosphere 2010;80:1353-9.

Cooper PF, Job GD, Green MB, Shutes RBE. Reed beds and constructed wetlands for wastewater treatment. UK: WRc Publications; 1996.

Corcoran J, Winter MJ, Tyler CR. Pharmaceuticals in the aquatic environment: a critical review of the evidence for health effects in fish. Crit Rev Toxicol 2010;40:287-304.

Cronk JK. Constructed wetlands to treat wastewater from dairy and swine operations: a review. Agric Ecosyst Environ 1996;58:97-114.

Davies LC, Pedro IS, Ferreira RA, Freire FG, Novais JM, Martins-Dias S. Constructed wetland treatment system in textile industry and sustainable development. Water Sci Technol 2008;58:2017-23.

Dettenmaier EM, Doucette WJ, Bugbee B. Chemical hydrophobicity and uptake by plant roots. Environ Sci Technol 2009;43:324-9.

Dietz AC, Schnoor JL. Advances in phytoremediation. Environ Health Perspect 2001;109: 163-8. 
Dordio AV, Carvalho AJP. Organic xenobiotics removal in constructed wetlands, with emphasis on the importance of the support matrix. J Hazard Mater 2013;252-253:272-92.

Dordio AV, Teimão J, Ramalho I, Carvalho AJP, Candeias AJE. Selection of a support matrix for the removal of some phenoxyacetic compounds in constructed wetlands systems. Sci Total Environ 2007:380:237-46.

Dordio A, Pinto J, Dias CB, Pinto AP, Carvalho AJP, Teixeira DM. Atenolol removal in microcosm constructed wetlands. Int J Environ Anal Chem 2009a;89:835-48.

Dordio AV, Duarte C, Barreiros M, Carvalho AJP, Pinto AP, da Costa CT. Toxicity and removal efficiency of pharmaceutical metabolite clofibric acid by Typha spp. - potential use for phytoremediation? Bioresour Technol 2009b;100:1156-61.

Dordio AV, Estêvão Candeias AJ, Pinto AP, Teixeira da Costa C, Palace Carvalho AJ. Preliminary media screening for application in the removal of clofibric acid, carbamazepine and ibuprofen by SSF-constructed wetlands. Ecol Eng 2009c;35:290-302.

Dordio A, Carvalho AJP, Teixeira DM, Dias CB, Pinto AP. Removal of pharmaceuticals in microcosm constructed wetlands using Typha spp. and LECA. Bioresour Techno 2010;101:886-92.

Dordio A, Ferro R, Teixeira D, Palace AJ, Pinto AP, Dias CMB. Study on the use of Typha spp. for the phytotreatment of water contaminated with ibuprofen. Int J Environ Anal Chem 2011a;91:654-67.

Dordio AV, Gonçalves P, Texeira D, Candeias AJ, Castanheiro JE, Pinto AP, et al. Pharmaceuticals sorption behaviour in granulated cork for the selection of a support matrix for a constructed wetlands system. Int J Environ Anal Chem 2011b;91:615-31.

Dordio AV, Belo M, Martins Teixeira D, Palace Carvalho AJ, Dias CMB, Picó Y, et al. Evaluation of carbamazepine uptake and metabolization by Typha spp., a plant with potential use in phytotreatment. Bioresour Technol 2011c;102:7827-34.

Dua M, Singh A, Sethunathan N, Johri A. Biotechnology and bioremediation: successes and limitations. Appl Microbiol Biotechnol 2002;59:143-52.

Farré MI, Pérez S, Kantiani L, Barceló D. Fate and toxicity of emerging pollutants, their metabolites and transformation products in the aquatic environment. TrAC Trends Ana Chem 2008;27:991-1007.

Fent K, Weston AA, Caminada D. Ecotoxicology of human pharmaceuticals. Aquat Toxico 2006;76:122-59.

Focazio MJ, Kolpin DW, Barnes KK, Furlong ET, Meyer MT, Zaugg SD, et al. A national reconnaissance for pharmaceuticals and other organic wastewater contaminants in the United States - II untreated drinking water sources. Sci Total Environ 2008;402:201-16.

Gagné $F$, Blaise C, André C. Occurrence of pharmaceutical products in a municipal effluen and toxicity to rainbow trout (Oncorhynchus mykiss) hepatocytes. Ecotoxicol Environ Saf 2006;64:329-36.

García J, Rousseau DPL, Morató J, Lesage E, Matamoros V, Bayona JM. Contaminant removal processes in subsurface-flow constructed wetlands: a review. Crit Rev Environ Sci Technol 2010;40:561-661.

Gross B, Montgomery-Brown J, Naumann A, Reinhard M. Occurrence and fate of pharmaceuticals and alkylphenol ethoxylate metabolites in an effluent-dominated river and wetland. Environ Toxicol Chem 2004;23:2074-83.

Haberl R, Grego S, Langergraber G, Kadlec RH, Cicalini AR, Dias SM, et al. Constructed wetlands for the treatment of organic pollutants. J Soils Sediments 2003;3:109-24.

Heberer T. Occurrence, fate, and removal of pharmaceutical residues in the aquatic environment: a review of recent research data. Toxicol Lett 2002a;131:5-17.

Heberer T. Tracking persistent pharmaceutical residues from municipal sewage to drinking water. J Hydrol 2002b;266:175-89.

Heberer T, Reddersen K, Mechlinski A. From municipal sewage to drinking water: fate and removal of pharmaceutical residues in the aquatic environment in urban areas. Water Sci Technol 2002;46:81-8.

Helt CD, Weber KP, Legge RL, Slawson RM. Antibiotic resistance profiles of representative wetland bacteria and faecal indicators following ciprofloxacin exposure in lab-scale constructed mesocosms. Ecol Eng 2012;39:113-22.

Hijosa-Valsero M, Matamoros V, Martín-Villacorta J, Bécares E, Bayona JM. Assessment of full-scale natural systems for the removal of PPCPs from wastewater in small communities. Water Res 2010a;44:1429-39.

Hijosa-Valsero M, Matamoros V, Sidrach-Cardona R, Martín-Villacorta J, Bécares E, Bayona JM. Comprehensive assessment of the design configuration of constructed wetland for the removal of pharmaceuticals and personal care products from urban wastewaters. Water Res 2010b;44:3669-78.

Hijosa-Valsero M, Fink G, Schlüsener MP, Sidrach-Cardona R, Martín-Villacorta J, Ternes T, et al. Removal of antibiotics from urban wastewater by constructed wetland optimization. Chemosphere 2011a;83:713-9.

Hijosa-Valsero M, Matamoros V, Pedescoll A, Martín-Villacorta J, Bécares E, García J, et al. Evaluation of primary treatment and loading regimes in the removal of pharmaceuticals and personal care products from urban wastewaters by subsurface-flow constructed wetlands. Int J Environ Anal Chem 2011b;91:632-53.

Hijosa-Valsero M, Matamoros V, Sidrach-Cardona R, Pedescoll A, Martín-Villacorta J, García J, et al. Influence of design, physico-chemical and environmental parameters on pharmaceuticals and fragrances removal by constructed wetlands. Water Sci Technol 2011c;63:2527-34.

Hussain SA, Prasher SO. Understanding the sorption of ionophoric pharmaceuticals in a treatment wetland. Wetlands 2011;31:563-71.

Hussain SA, Prasher SO, Patel R. Removal efficiency of horizontal subsurface flow wetlands for veterinary pharmaceuticals. Trans ASABE 2011;54:2037-46.

Hussain SA, Prasher SO, Patel RM. Removal of ionophoric antibiotics in free water surface constructed wetlands. Ecol Eng 2012;41:13-21.

Jelic A, Gros M, Ginebreda A, Cespedes-Sánchez R, Ventura F, Petrovic M, et al. Occurrence, partition and removal of pharmaceuticals in sewage water and sludge during wastewater treatment. Water Res 2011;45:1165-76.

Jiang JQ Zhou Z, Sharma VK. Occurrence, transportation, monitoring and treatment of emerging micro-pollutants in waste water - a review from global views. Microchem J 2013;110:292-300
Jones $\mathrm{OAH}$, Voulvoulis N, Lester JN. Human pharmaceuticals in the aquatic environment a review. Environ Technol 2001;22:1383-94.

Jones OAH, Voulvoulis N, Lester JN. Aquatic environmental assessment of the top 25 English prescription pharmaceuticals. Water Res 2002;36:5013-22.

Jones OAH, Voulvoulis N, Lester JN. Potential ecological and human health risks associated with the presence of pharmaceutically active compounds in the aquatic environment. Crit Rev Toxicol 2004;34:335-50.

Jones $\mathrm{OAH}$, Voulvoulis N, Lester JN. The occurrence and removal of selected pharmaceutical compounds in a sewage treatment works utilising activated sludge treatment. Environ Pollut 2007;145:738-44

Joss A, Zabczynski S, Göbel A, Hoffmann B, Löffler D, McArdell CS, et al. Biological degradation of pharmaceuticals in municipal wastewater treatment: proposing a classification scheme. Water Res 2006;40:1686-96.

Kadlec RH, Wallace SD. Treatment wetlands. Vol 2nd ed. Boca Raton, FL: CRC Press; 2009.

Kasprzyk-Hordern B, Dinsdale RM, Guwy AJ. The removal of pharmaceuticals, personal care products, endocrine disruptors and illicit drugs during wastewater treatment and its impact on the quality of receiving waters. Water Res 2009;43:363-80.

Kim SD, Cho J, Kim IS, Vanderford BJ, Snyder SA. Occurrence and removal of pharmaceuticals and endocrine disruptors in South Korean surface, drinking, and waste waters. Water Res 2007;41:1013-21.

Kimura K, Hara H, Watanabe Y. Elimination of selected acidic pharmaceuticals from municipal wastewater by an activated sludge system and membrane bioreactors. Environ Sci Technol 2007;41:3708-14.

Klamerth N, Rizzo L, Malato S, Maldonado MI, Agüera A, Fernández-Alba AR. Degradation of fifteen emerging contaminants at $\mu \mathrm{g}$ L- 1 initial concentrations by mild solar photo-Fenton in MWTP effluents. Water Res 2010;44:545-54.

Kosma CI, Lambropoulou DA, Albanis TA. Occurrence and removal of PPCPs in municipal and hospital wastewaters in Greece. J Hazard Mater 2010;179:804-17.

Kurwadkar ST, Adams CD, Meyer MT, Kolpin DW. Effects of sorbate speciation on sorption of selected sulfonamides in three loamy soils. J Agric Food Chem 2007;55:1370-6.

Lee S, Kang SI, Lim JL, Huh YJ, Kim KS, Cho J. Evaluating controllability of pharmaceuticals and metabolites in biologically engineered processes, using corresponding octanolwater distribution coefficient. Ecol Eng 2011;37:1595-600.

Lertpaitoonpan W, Ong SK, Moorman TB. Effect of organic carbon and pH on soil sorption of sulfamethazine. Chemosphere 2009;76:558-64.

Li M, Zhou Q, Tao M, Wang Y, Jiang L, Wu Z. Comparative study of microbial community structure in different filter media of constructed wetland. J Environ Sci 2010;22:127-33.

Li Y, Jindal R, Choi K, Kho YL, de Bullen PG. Pharmaceutical residues in wastewater treatment plants and surface waters in Bangkok. J Hazard Toxic Radioact Waste 2011;16:88-91.

Lin AYC, Yu TH, Lateef SK. Removal of pharmaceuticals in secondary wastewater treatment processes in Taiwan. J Hazard Mater 2009;167:1163-9.

Lindqvist N, Tuhkanen T, Kronberg L. Occurrence of acidic pharmaceuticals in raw and treated sewages and in receiving waters. Water Res 2005;39:2219-28.

Liu L, Liu C, Zheng J, Huang X, Wang Z, Liu Y, et al. Elimination of veterinary antibiotics and antibiotic resistance genes from swine wastewater in the vertical flow constructed wetlands. Chemosphere 2013a;91:1088-93.

Liu L, Y-h Liu, Liu C-X, Wang Z, Dong J, Zhu G-F, et al. Potential effect and accumulation of veterinary antibiotics in Phragmites australis under hydroponic conditions. Ecol Eng 2013b;53:138-43.

Llorens E, Matamoros V, Domingo V, Bayona JM, García J. Water quality improvement in a full-scale tertiary constructed wetland: effects on conventional and specific organic contaminants. Sci Total Environ 2009;407:2517-24.

Martínez F, López-Muñoz MJ, Aguado J, Melero JA, Arsuaga J, Sotto A, et al. Coupling membrane separation and photocatalytic oxidation processes for the degradation of pharmaceutical pollutants. Water Res 2013. (in press).

Matamoros V, Bayona JM. Elimination of pharmaceuticals and personal care products in subsurface flow constructed wetlands. Environ Sci Technol 2006;40:5811-6.

Matamoros V, Salvadó V. Evaluation of the seasonal performance of a water reclamation pond-constructed wetland system for removing emerging contaminants. Chemosphere 2012;86:111-7.

Matamoros V, García J, Bayona JM. Behavior of selected pharmaceuticals in subsurface flow constructed wetlands: a pilot-scale study. Environ Sci Technol 2005;39:5449-54

Matamoros V, Arias C, Brix H, Bayona JM. Removal of pharmaceuticals and personal care products (PPCPs) from urban wastewater in a pilot vertical flow constructed wetland and a sand filter. Environ Sci Technol 2007:41:8171-7.

Matamoros V, Caselles-Osorio A, García J, Bayona JM. Behaviour of pharmaceutical products and biodegradation intermediates in horizontal subsurface flow constructed wetland. A microcosm experiment. Sci Total Environ 2008a;394:171-6.

Matamoros V, García J, Bayona JM. Organic micropollutant removal in a full-scale surface flow constructed wetland fed with secondary effluent. Water Res 2008b;42:653-60.

Matamoros V, Arias C, Brix H, Bayona JM. Preliminary screening of small-scale domestic wastewater treatment systems for removal of pharmaceutical and personal care products. Water Res 2009a;43:55-62.

Matamoros V, Hijosa M, Bayona JM. Assessment of the pharmaceutical active compounds removal in wastewater treatment systems at enantiomeric level. Ibuprofen and naproxen. Chemosphere 2009b;75:200-5.

Matamoros V, Arias CA, Nguyen LX, Salvadó V, Brix H. Occurrence and behavior of emerging contaminants in surface water and a restored wetland. Chemosphere 2012a;88: $1083-9$

Matamoros V, Nguyen LX, Arias CA, Salvadó V, Brix H. Evaluation of aquatic plants for removing polar microcontaminants: a microcosm experiment. Chemosphere 2012b;88: 1257-64.

Matsuo H, Sakamoto H, Arizono K, Shinohara R. Behavior of pharmaceuticals in waste water treatment plant in Japan. Bull Environ Contam Toxicol 2011;87:31-5.

Maurer M, Escher BI, Richle P, Schaffner C, Alder AC. Elimination of $\beta$-blockers in sewage treatment plants. Water Res 2007;41:1614-22. 
Mestre AS, Pires J, Nogueira JMF, Parra JB, Carvalho AP, Ania CO. Waste-derived activated carbons for removal of ibuprofen from solution: role of surface chemistry and pore structure. Bioresour Technol 2009;100:1720-6.

Miège $C$, Choubert JM, Ribeiro L, Eusèbe M, Coquery M. Fate of pharmaceuticals and personal care products in wastewater treatment plants - conception of a database and first results. Environ Pollut 2009; 157:1721-6.

Molinos-Senante M, Reif R, Garrido-Baserba M, Hernández-Sancho F, Omil F, Poch M, et al. Economic valuation of environmental benefits of removing pharmaceutical and personal care products from WWTP effluents by ozonation. Sci Total Environ 2013;461-462:409-15.

Naddeo V, Belgiorno V, Ricco D, Kassinos D. Degradation of diclofenac during sonolysis, ozonation and their simultaneous application. Ultrason Sonochem 2009;16:790-4.

Pal A, Gin KY-H, Lin AY-C, Reinhard M. Impacts of emerging organic contaminants on freshwater resources: review of recent occurrences, sources, fate and effects. Sci Total Environ 2010;408:6062-9.

Park N, Vanderford BJ, Snyder SA, Sarp S, Kim SD, Cho J. Effective controls of micropollutants included in wastewater effluent using constructed wetlands under anoxic condition. Ecol Eng 2009;35:418-23.

Paxéus N. Removal of selected non-steroidal anti-inflammatory drugs (NSAIDs), gemfibrozil, carbamazepine, $\beta$-blockers, trimethoprim and triclosan in conventional wastewater treatment plants in five EU countries and their discharge to the aquatic environment. Water Sci Technol 2004;50:253-60.

Pei Z, Kong J, Shan XQ Wen B. Sorption of aromatic hydrocarbons onto montmorillonite as affected by norfloxacin. J Hazard Mater 2012;203-204:137-44.

Petrie B, McAdam EJ, Scrimshaw MD, Lester JN, Cartmell E. Fate of drugs during wastewater treatment. TrAC Trends Anal Chem 2013:49:145-59.

Pomati F, Castiglioni S, Zuccato E, Fanelli R, Vigetti D, Rossetti C, et al. Effects of a complex mixture of therapeutic drugs at environmental levels on human embryonic cells. Environ Sci Technol 2006;40:2442-7.

Ranieri E, Verlicchi P, Young TM. Paracetamol removal in subsurface flow constructed wetlands. J Hydrol 2011;404:130-5.

Reddy KR, DeLaune RD. Biogeochemistry of wetlands: science and applications. Boca Raton, FL: CRC Press; 2008.

Reif R, Besancon A, Le Corre K, Jefferson B, Lema JM, Omil F. Comparison of PPCPs removal on a parallel-operated MBR and AS system and evaluation of effluent post-treatment on vertical flow reed beds. Water Sci Technol 2011;63:2411-7.

Reinhold D, Vishwanathan S, Park JJ, Oh D, Michael Saunders F. Assessment of plant-driven removal of emerging organic pollutants by duckweed. Chemosphere 2010;80:687-92.

Repice C, Grande MD, Maggi R, Pedrazzani R. Licit and illicit drugs in a wastewater treatment plant in Verona, Italy. Sci Total Environ 2013;463-464:27-34.

Reyes-Contreras C, Matamoros V, Ruiz I, Soto M, Bayona JM. Evaluation of PPCPs removal in a combined anaerobic digester-constructed wetland pilot plant treating urban wastewater. Chemosphere 2011;84:1200-7.

Reyes-Contreras C, Hijosa-Valsero M, Sidrach-Cardona R, Bayona JM, Bécares E. Temporal evolution in PPCP removal from urban wastewater by constructed wetlands of different configuration: a medium-term study. Chemosphere 2012;88:161-7.

Richardson SD. Water analysis: emerging contaminants and current issues. Anal Chem 2007;79:4295-323.

Rossner A, Snyder SA, Knappe DRU. Removal of emerging contaminants of concern by alternative adsorbents. Water Res 2009;43:3787-96.

Salomo S, Münch C, Röske I. Evaluation of the metabolic diversity of microbial communities in four different filter layers of a constructed wetland with vertical flow by Biolog TM analysis. Water Res 2009;43:4569-78.

Samaras VG, Stasinakis AS, Mamais D, Thomaidis NS, Lekkas TD. Fate of selected pharmaceuticals and synthetic endocrine disrupting compounds during wastewater treatment and sludge anaerobic digestion. J Hazard Mater 2013;244-245:259-67.

Santos JL, Aparicio I, Callejón M, Alonso E. Occurrence of pharmaceutically active compounds during 1-year period in wastewaters from four wastewater treatment plants in Seville (Spain). J Hazard Mater 2009;164:1509-16.

Santos LHMLM, Gros M, Rodriguez-Mozaz S, Delerue-Matos C, Pena A, Barceló D, et al. Contribution of hospital effluents to the load of pharmaceuticals in urban wastewaters: identification of ecologically relevant pharmaceuticals. Sci Total Environ 2013;461-462:302-16

Sassman SA, Lee LS. Sorption and degradation in soils of veterinary ionophore antibiotics: monensin and lasalocid. Environ Toxicol Chem 2007;26:1614-21.

Saussereau E, Lacroix C, Guerbet M, Cellier D, Spiroux J, Goullé JP. Determination of levels of current drugs in hospital and urban wastewater. Bull Environ Contam Toxicol 2013;91:171-6.

Schröder P, Collins C. Conjugating enzymes involved in xenobiotic metabolism of organic xenobiotics in plants. Int J Phytoremediation 2002:4:247-65.

Seffernick JL, Wackett LP. Rapid evolution of bacterial catabolic enzymes: a case study with atrazine chlorohydrolase. Biochemistry 2001;40:12747-53.

Sim W-J, Lee J-W, Oh J-E. Occurrence and fate of pharmaceuticals in wastewater treatment plants and rivers in Korea. Environ Pollut 2010;158:1938-47.

Singh KP, Malik A, Sinha S, Ojha P. Liquid-phase adsorption of phenols using activated carbons derived from agricultural waste material. J Hazard Mater 2008;150:626-41.

Sipma J, Osuna B, Collado N, Monclús H, Ferrero G, Comas J, et al. Comparison of removal of pharmaceuticals in MBR and activated sludge systems. Desalination 2010;250:653-9.

Stottmeister U, Wießner A, Kuschk P, Kappelmeyer U, Kästner M, Bederski O, et al. Effects of plants and microorganisms in constructed wetlands for wastewater treatment Biotechnol Adv 2003;22:93-117.

Sundaravadivel M, Vigneswaran S. Constructed wetlands for wastewater treatment. Crit. Rev. Environ. Sci. Technol. 2001;31:351-409.

Ternes TA. Occurrence of drugs in German sewage treatment plants and rivers. Water Res 1998;32:3245-60.
Ternes T, Bonerz M, Schmidt T. Determination of neutral pharmaceuticals in wastewate and rivers by liquid chromatography-electrospray tandem mass spectrometry. J Chromatogr A 2001;938:175-85.

Ternes TA, Joss A, Siegrist H. Scrutinizing pharmaceuticals and personal care products in wastewater treatment. Environ Sci Technol 2004:38:392A-9A.

Ternes TA, Bonerz M, Herrmann N, Teiser B, Andersen HR. Irrigation of treated wastewater in Braunschweig, Germany: an option to remove pharmaceuticals and musk fragrances. Chemosphere 2007;66:894-904.

Tewari S, Jindal R, Kho YL, Eo S, Choi K. Major pharmaceutical residues in wastewater treatment plants and receiving waters in Bangkok, Thailand, and associated ecological risks. Chemosphere 2013;91:697-704.

Tolls J. Sorption of veterinary pharmaceuticals in soils: a review. Environ Sci Technol 2001;35:3397-406.

Trinh T, Van Den Akker B, Stuetz RM, Coleman HM, Le-Clech P, Khan SJ. Removal of trace organic chemical contaminants by a membrane bioreactor. Water Sci Technol 2012;66:1856-63.

Truu M, Juhanson J, Truu J. Microbial biomass, activity and community composition in constructed wetlands. Sci Total Environ 2009;407:3958-71.

Uslu MO, Jasim S, Arvai A, Bewtra J, Biswas N. A survey of occurrence and risk assessment of pharmaceutical substances in the Great Lakes Basin. Ozone Sci Eng 2013;35:249-62.

Vasudevan D, Bruland GL, Torrance BS, Upchurch VG, MacKay AA. pH-dependent ciprofloxacin sorption to soils: interaction mechanisms and soil factors influencing sorption. Geoderma 2009;151:68-76.

Verlicchi P, Al Aukidy M, Zambello E. Occurrence of pharmaceutical compounds in urban wastewater: removal, mass load and environmental risk after a secondary treatment-a review. Sci Total Environ 2012;429:123-55

Verlicchi P, Galletti A, Petrovic M, Barceló D, Al Aukidy M, Zambello E. Removal of selected pharmaceuticals from domestic wastewater in an activated sludge system followed by a horizontal subsurface flow bed - analysis of their respective contributions. Sci Total Environ 2013;454-455:411-25.

Vidal-Dorsch DE, Bay SM, Maruya K, Snyder SA, Trenholm RA, Vanderford BJ. Contaminants of emerging concern in municipal wastewater effluents and marine receiving water. Environ Toxicol Chem 2012;31:2674-82.

Vieno NM, Tuhkanen T, Kronberg L. Seasonal variation in the occurrence of pharmaceuticals in effluents from a sewage treatment plant and in the recipient water. Environ Sci Technol 2005;39:8220-6.

Vieno N, Tuhkanen T, Kronberg L. Elimination of pharmaceuticals in sewage treatment plants in Finland. Water Res 2007;41:1001-12.

Vymazal J. The use constructed wetlands with horizontal sub-surface flow for various types of wastewater. Ecol Eng 2009;35:1-17.

Vymazal J, Brix H, Cooper PF, Green MB, Haberl R. Constructed wetlands for wastewater treatment in Europe. Leiden, Netherlands: Backhuys Publishers; 1998.

Weber KP, Mitzel MR, Slawson RM, Legge RL. Effect of ciprofloxacin on microbiological development in wetland mesocosms. Water Res 2011;45:3185-96.

Xian Q, Hu L, Chen H, Chang Z, Zou H. Removal of nutrients and veterinary antibiotics from swine wastewater by a constructed macrophyte floating bed system. J Environ Manage 2010;91:2657-61.

Xu J, Wu L, Chang AC. Degradation and adsorption of selected pharmaceuticals and personal care products (PPCPs) in agricultural soils. Chemosphere 2009;77:1299-305.

Yang Y, Fu J, Peng H, Hou L, Liu M, Zhou JL. Occurrence and phase distribution of selected pharmaceuticals in the Yangtze Estuary and its coastal zone. J Hazard Mater 2011;190:588-96.

Yu Y, Liu Y, Wu L. Sorption and degradation of pharmaceuticals and personal care products (PPCPs) in soils. Environ. Sci. Pollut. Res. 2013:1-7.

Zarate Jr FM, Schulwitz SE, Stevens KJ, Venables BJ. Bioconcentration of triclosan, methyl-triclosan, and triclocarban in the plants and sediments of a constructed wetland. Chemosphere 2012:88:323-9.

Zhang Y, Geißen S-U, Gal C. Carbamazepine and diclofenac: removal in wastewater treatment plants and occurrence in water bodies. Chemosphere 2008;73:1151-61.

Zhang DQ, Tan SK, Gersberg RM, Sadreddini S, Zhu J, Tuan NA. Removal of pharmaceutical compounds in tropical constructed wetlands. Ecol Eng 2011;37:460-4.

Zhang DQ Gersberg RM, Hua T, Zhu J, Tuan NA, Tan SK. Pharmaceutical removal in tropica subsurface flow constructed wetlands at varying hydraulic loading rates. Chemosphere 2012a;87:273-7.

Zhang DQ, Gersberg RM, Zhu J, Hua T, Jinadasa KBSN, Tan SK. Batch versus continuous feeding strategies for pharmaceutical removal by subsurface flow constructed wetland. Environ Pollut 2012b;167:124-31.

Zhang DQ, Hua T, Gersberg RM, Zhu J, Ng WJ, Tan SK. Fate of diclofenac in wetland mesocosms planted with Scirpus validus. Ecol Eng 2012c;49:59-64.

Zhang DQ, Hua T, Gersberg RM, Zhu J, Ng WJ, Tan SK. Fate of caffeine in mesocosms wetland planted with Scirpus validus. Chemosphere 2013a;90:1568-72.

Zhang J, Chang VWC, Giannis A, Wang J-Y. Removal of cytostatic drugs from aquatic environment: a review. Sci Total Environ 2013b;445-446:281-98.

Zhu G, Jetten MSM, Kuschk P, Ettwig KF, Yin C. Potential roles of anaerobic ammonium and methane oxidation in the nitrogen cycle of wetland ecosystems. Appl Microbiol Biotechnol 2010;86:1043-55.

Zhu G, Wang S, Feng X, Fan G, Jetten MSM, Yin C. Anammox bacterial abundance, biodiversity and activity in a constructed wetland. Environ Sci Technol 2011:45:9951-8.

Zhu G, Wang S, Wang W, Wang Y, Zhou L, Jiang B, et al. Hotspots of anaerobic ammonium oxidation at land-freshwater interfaces. Nat Geosci 2013;6:103-7.

Zuccato E, Castiglioni S, Fanelli R, Reitano G, Bagnati R, Chiabrando C, et al. Pharmaceuticals in the environment in Italy: causes, occurrence, effects and control. Environ. Sci. Pollut. Res. 2006;13:15-21.

Zwiener C, Seeger S, Glauner T, Frimmel FH. Metabolites from the biodegradation of pharmaceutical residues of ibuprofen in biofilm reactors and batch experiments. Anal Bioanal Chem 2002:372:569-75. 